\title{
Assessing proportionality assumption in the adjacent category logistic regression model
}

\author{
Anil Dolgun ${ }^{*} \uparrow$ And Osman SARACBasi
}

Ordinal logistic regression models are classified as either proportional odds models, continuation ratio models or adjacent category models. The common model assumption of these models is that the log odds do not depend on the outcome category. This assumption is also known as the "proportionality" or "parallel logits" assumption. Nonproportional and partial proportional models are proposed for the proportional odds and continuation ratio model. The non-proportional and the partial proportional versions of the adjacent category model are also feasible. Prior to fitting any of the ordinal logistic regression models, it is important to check whether the assumption of proportionality is satisfied by each independent variable. In the proportional odds model, the proportional odds assumption is checked by Brant's Wald test statistic, and the standard Wald test statistic can be used in the continuation ratio model. However there is no valid approach to test whether the proportionality assumption is satisfied by each independent variable in the adjacent category model. The aim of the study is to determine the variables in the adjacent category model that violate the proportionality assumption. For this purpose, a Wald test is proposed for testing the proportionality assumption in the adjacent category model. The validity of the proposed test is examined under $H_{0}$ with a Monte Carlo simulation study. Moreover, the proposed method is compared with the likelihood ratio test in terms of type I error rate and power under different scenarios.

AMS 2000 SUBJECT Classifications: Primary 62J12, 62F05; secondary 62P10, 62P25.

KEYWORDs AND PHRASEs: Ordinal logistic regression, Proportionality assumption, Adjacent category model, Odds ratio, Wald test, Likelihood ratio test.

\section{INTRODUCTION}

The logistic regression analysis is a useful way of describing the relationship between one or more independent variables and a categorical response variable. Logistic regression models are categorized according to the type of categorical response variable as follows: binary logistic regression model,

* Corresponding author.

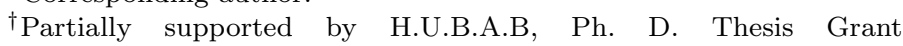
09.T01.101.001. multinomial logistic regression model, and ordinal logistic regression models [1]. The binary logistic regression model is used to model the binary response variable, whereas the multinomial logistic regression is a simple extension of the binary logistic regression model where the response variable has more than two unordered categories.

Response variables can also be measured on an ordinal scale such as extent of disease (none, some, severe), cardiac risk levels (low, medium and high risk) and grade of a tumour (grade I-II-III). Outcome data collected using an ordinal scale are sometimes analyzed with a binary logistic regression by creating dichotomies among the level of the outcome variable $[2,3]$. Although such approaches are not incorrect, they often result in a loss of information due to collapsing (or ignoring) some categories of the response, typically resulting in a considerable loss of statistical power [4]. Additionally, the multinomial logistic regression model is often used for modelling multiple category ordinal outcomes. This analysis, however, would not take into account the ordinal nature of the outcome [1]. Therefore, ordinal logistic regression models are used to model the relationship between independent variables and an ordinal response variable when the response variable has a natural ordering.

A number of ordinal logistic regression models for analyzing ordinal outcome data have been proposed [5-14], and the application of these methods are widely used, especially in epidemiological and biomedical research [15-20].

Probably the most frequently used ordinal logistic regression model is the proportional odds model of McCullagh because of the simplicity of its interpretation [6]. The continuation ratio model may be best suited in situations where the individual categories of the response variable are of intrinsic interest and are not merely arbitrary groupings of an underlying continuous variable [15]. Adjacent category models are often used due to their close connection to log-linear models [9].

Although these ordinal logistic regression models have different structures and strategies for constructing logits, they share the same model assumption. The common model assumption of these models is that the log odds do not depend on the outcome category. This assumption is also known as the "proportionality" or "parallel logits" assumption [1]. Unfortunately, this basic and strict assumption frequently does not hold [21].

When the assumption of parallel logits is rejected for any of the ordinal logistic regression models, alternative models 
should be considered that do not impose the constraint of parallel logits. For every ordinal logistic regression model, there are two alternative models to use in cases of nonproportional odds. Non-proportional (e.g., relaxing the proportionality assumption for all of the variables in a model) and partial proportional models (e.g., relaxing the proportionality assumption only for some) are proposed for the proportional odds model $[14,22,23]$ and the continuation ratio model $[24,25]$. The non-proportional and the partial proportional versions of the adjacent category model are also feasible.

Prior to fitting any of ordinal logistic regression models, it is important to check whether the assumption of proportionality is satisfied by each independent variable. In the proportional odds model, the proportional odds assumption is checked by Brant's Wald test statistic [26], and it is performed using the standard Wald test statistic [1] in the continuation ratio model. However there is no valid approach to test whether the proportionality assumption is satisfied by each independent variable in the adjacent category model.

The aim of this study is to determine the variables in the adjacent category model that violate the proportionality assumption. For this purpose, a Wald test is proposed for testing the proportionality assumption in the adjacent category model. With use of the proposed test statistic, the variables in the adjacent category model that do not obey the proportionality assumption can be determined, and they can be modelled as non-proportional in the partial proportional adjacent category model.

Through Sections 2 and 3, the multinomial and ordinal logistic regression models are presented. Methods used for checking the proportional odds assumption are given in Section 4. In Section 5, we adapt the Wald test of Brant in order to test the proportionality assumption in the adjacent category model. The validity of the proposed test is examined under $H_{0}$ with a Monte Carlo simulation study. The proposed method is compared with the likelihood ratio test in terms of type I error rate and power under different scenarios in Section 6, and the simulation results are given in Section 7. Additionally, an example of the proposed test of proportionality calculations in the adjacent category model is given in Section 8. Finally in Section 9, we discuss our findings.

\section{THE MULTINOMIAL LOGISTIC REGRESSION MODEL}

The multinomial logistic regression model (MLRM) is a simple extension of the binary logistic regression model in which the response variable has more than two unordered categories. This model can be fitted by simultaneously estimating binary logistic regression models for all possible comparisons of the outcome category with a baseline category. This is why MLRM is often called the "baseline category logistic regression model".
Let $Y$ denote the outcome variable and $k$ denote the outcome variable category where $k=0,1, \ldots, K$. The probability that the outcome is equal to $k$, conditional on a vector $\mathbf{x}$ of $j$ covariates $(j=1,2, \ldots, J)$ is denoted by $P(Y=k \mid \mathbf{x})=\phi_{k}(\mathbf{x})$. In the multinomial logistic regression model, generally the first category of the outcome $(k=0)$ is set to be the baseline, and the probability of the baseline, conditional on $\mathbf{x}$, is denoted by $P(Y=0 \mid \mathbf{x})=\phi_{0}(\mathbf{x})$. Using the multinomial logit link, the MLRM can be expressed in terms of logit (log odds) as:

(1)

$g_{k}(\mathbf{x})=\ln \left[\frac{P(Y=k \mid \mathbf{x})}{P(Y=0 \mid \mathbf{x})}\right]=\beta_{0 k}+\mathbf{x}^{\prime} \beta_{k} \quad k=1,2, \ldots, K$.

Additionally, the probabilities in Equation (1) are equal to:

$$
\begin{aligned}
& P(Y=0 \mid \mathbf{x})=\frac{1}{1+\sum_{i=1}^{K} e^{\beta_{0 i}+\mathbf{x}^{\prime} \boldsymbol{\beta}_{i}}}, \\
& P(Y=k \mid \mathbf{x})=\frac{e^{\beta_{0 k}+\mathbf{x}^{\prime} \boldsymbol{\beta}_{k}}}{1+\sum_{i=1}^{K} e^{\beta_{0 i}+\mathbf{x}^{\prime} \boldsymbol{\beta}_{i}}},
\end{aligned}
$$

and

$$
\sum_{k=0}^{K} P(Y=k \mid \mathbf{x})=1
$$

In Equation (1), $\beta_{0 k}$ is the constant term, and $\beta_{k}$ is the regression coefficient vector in the $k$ th logit. The benefit of using MLRM is that it models the odds of each category relative to a baseline category as a function of covariates. The parameter and the standard error estimates, simultaneously satisfying $K$ multinomial logits can be estimated using the maximum likelihood estimation method. Further details can be found in McCullagh and Nelder's book [11, p. 171-174].

MLRM is often used when the dependent variable is ordinal. If the dependent variable is ordinal and a model for nominal variables is used, there is a loss of efficiency because this information (ordinality) is ignored [21]. In such cases, one should consider using ordinal logistic regression models instead. Additionally, before considering methods for ordinal outcomes, it is important to note that the fact that the variables can be ordered does not imply that the variable should be analyzed as ordinal. A variable might be ordered when considered for one purpose but can be unordered when used for another purpose. McCullagh and Nelder [11, p. 151] make this point with the example of colours. While colours can be arranged according to the electromagnetic spectrum, this doesn't imply that this ordering is appropriate for all purposes. For example, when selecting a car, a customer doesn't prefer colours in an order that moves around the colour wheel from red, to orange, to yellow, and so on. 


\section{ORDINAL LOGISTIC REGRESSION MODELS}

When we move to an ordinal model, there are many alternative models. We must decide what outcomes to compare and what the most reasonable model is for the logit. Ordinal logistic regression models have basically three members, the proportional odds model, continuation ratio model, and adjacent category model. These models assume that the log odds do not depend on the outcome category. Other models are derivations of them, relaxing the proportionality assumption for some or for all of the independent variables in a model.

\subsection{The proportional odds model}

Probably the most frequently used ordinal logistic regression model is the proportional odds model (POM) because of the simplicity of its interpretation [6]. The POM uses cumulative logits while building the model. The cumulative logits are defined as

$$
\operatorname{logit}[Y \leq k \mid \mathbf{x}]=\ln \left[\frac{P(Y \leq k \mid \mathbf{x})}{1-P(Y \leq k \mid \mathbf{x})}\right] .
$$

A model that simultaneously uses all cumulative logits for all $k=0,1, \ldots, K-1$ is called the POM and is given as follows:

$$
c_{k}(\mathbf{x})=\ln \left[\frac{P(Y \leq k \mid \mathbf{x})}{1-P(Y \leq k \mid \mathbf{x})}\right]=\tau_{k}-\mathbf{x}^{\prime} \boldsymbol{\beta} .
$$

The cumulative probability in Equation (6) is defined as

$$
P(Y \leq k \mid \mathbf{x})=\frac{e^{\tau_{k}-\mathbf{x}^{\prime} \boldsymbol{\beta}}}{1+e^{\tau_{k}-\mathbf{x}^{\prime} \boldsymbol{\beta}}},
$$

and the actual category probability is defined as

$$
\begin{aligned}
P(Y=k \mid \mathbf{x}) & =P(Y \leq k \mid \mathbf{x})-P(Y \leq k-1 \mid \mathbf{x}) \\
& =\frac{e^{\tau_{k}-\mathbf{x}^{\prime} \boldsymbol{\beta}}}{1+e^{\tau_{k}-\mathbf{x}^{\prime} \boldsymbol{\beta}}}-\frac{e^{\tau_{k-1}-\mathbf{x}^{\prime} \boldsymbol{\beta}}}{1+e^{\tau_{k-1}-\mathbf{x}^{\prime} \boldsymbol{\beta}}} .
\end{aligned}
$$

Note that in POM, each logit has its own intercept $\left(\tau_{k}\right)$, but the regression coefficient $(\boldsymbol{\beta})$ does not depend on $k$, implying that the model assumes that the relationship between $x_{i}$ and $\mathrm{Y}$ is independent of $k$. McCullagh calls this assumption "the proportional odds" or "the parallel regression" assumption. The POM in Equation (6) has some attractive features. Because the regression parameters are invariant to the outcome category, the odds ratios are the same over the $K$ logits, and the common log odds ratio provides a single estimate of the log odds ratio over the cut-off points. This estimate is not a weighted average of the cut-off point-specific log odds ratios [27], but it is the optimum estimate obtained using the maximum likelihood methods. In the POM, cumulative logits are simultaneously modelled using the maximum likelihood estimation method.
Prior to fitting a POM, it is important to check whether the assumption of proportionality is satisfied by each variable in the model. Methods used for checking the proportionality assumption are given in Section 4. In cases where the proportional odds assumption is inadequate, alternative strategies to improve the fit include (i) trying different link functions such as probit or log-log, (ii) adding additional terms such as interactions to the model, (iii) generalizing the model by adding dispersion parameters [11], (iv) using a multinomial logistic regression instead, and (v) permitting separate effects for each logit for some or all of the covariates in a model. Through Sections 3.2 and 3.3, nonproportional and partial proportional odds models that allow separate effects for each logit for all or some of the variables are presented.

\subsection{The non-proportional odds model}

The non-proportional odds model (NPOM) relaxes the assumption of proportional odds by allowing the effect of the covariates to vary with the point where the categories of the response variable are dichotomized. For $k=0,1, \ldots, K-1$, the NPOM is given as follows [22, 23]:

$$
c_{k}^{N P}(\mathbf{x})=\ln \left[\frac{P(Y \leq k \mid \mathbf{x})}{1-P(Y \leq k \mid \mathbf{x})}\right]=\tau_{k}-\mathbf{x}^{\prime} \boldsymbol{\beta}_{k} .
$$

In NPOM, each logit has its own intercept $\left(\tau_{k}\right)$ and regression coefficient $\left(\boldsymbol{\beta}_{k}\right)$. Thus, it relaxes the proportional assumption for all covariates in the model. If only some of the covariates violate this assumption, then a more parsimonious model should be considered.

\subsection{The partial proportional odds model}

Under the partial proportional odds model (PPOM) [14], two sets of regression coefficients are estimated: one set having proportional odds and the second set having nonproportional odds. For $k=0,1, \ldots, K-1$, this model has the form:

$$
c_{k}^{P P}(\mathbf{x})=\ln \left[\frac{P(Y \leq k \mid \mathbf{x})}{1-P(Y \leq k \mid \mathbf{x})}\right]=\tau_{k}-\mathbf{x}^{\prime} \boldsymbol{\beta}-\mathbf{T}^{\prime} \boldsymbol{\gamma}_{k} .
$$

In Equation (10), $\mathbf{x}$ is a $p \times 1$ vector of covariates that maintain the proportional odds property, and $\mathbf{T}$ is a $q \times 1$ vector of covariates that are non-proportional. If each covariate violates the proportionality, then the model in Equation (10) is equivalent to the NPOM in Equation (9). The independent variables (and thus the corresponding regression coefficients) which meet or do not meet the proportionality assumption in the PPOM, can be determined with the Brant's Wald test statistic given in Section 4.2.

\subsection{The continuation ratio model}

Feinberg [10] proposed an alternative model for the analysis of ordinal categorical data. The continuation ratio model $(\mathrm{CRM})$ is based on the conditional probabilities of being in 
a category $(Y=k)$ among all subjects who are in higher categories $(Y>k)$ and is given as:

(11)

$r_{k}(\mathbf{x})=\ln \left[\frac{P(Y=k \mid \mathbf{x})}{P(Y>k \mid \mathbf{x})}\right]=\theta_{k}-\mathbf{x}^{\prime} \beta \quad k=0,1, \ldots, K-1$.

The CRM is a simple decomposition of a multinomial distribution [28]. Using this property, multinomial likelihood for the model can be decomposed into a series of conditionally independent binary logistic regressions [29] and a natural way of modelling the continuation ratio logits would be to consider each ordinal response as a series of conditionally independent binary responses, each of which may be modelled via binary logistic regression. Each fit is based on a binary outcome $y_{k}^{*}$, defined as

$$
y_{k}^{*}= \begin{cases}1 & \text { if } Y=k \\ 0 & \text { if } Y>k \\ \text { Missing } & \text { else }\end{cases}
$$

for $k=0,1,2$. Wolfe [29] developed a command for fitting the continuation ratio model in STATA software. It is also possible to define the CRM in terms of $Y=k$ versus $Y<k$ for $k=1,2, \ldots, K$. Unfortunately, the results obtained from these two parameterizations are not equivalent. In other words, unlike the POM, the CRM is neither preserved by a reversal nor the collapsibility of categories [4].

The test of the proportional odds assumption in the CRM is performed using the likelihood ratio or the Wald test statistic. Details and an example can be found in Hosmer and Lemeshow [1, p. 297]. In cases where the proportional odds assumption does not hold for some or all of the covariates in the CRM, then alternative models should be considered. Through Sections 3.5 and 3.6, alternative models which accommodate this assumption are considered.

\subsection{The non-proportional continuation ratio model}

The non-proportional continuation ratio model (NPCRM) relaxes the assumption of proportional odds for all covariates in the model as follows [1, 24]:

(13)

$r_{k}^{N P}(\mathbf{x})=\ln \left[\frac{P(Y=k \mid \mathbf{x})}{P(Y>k \mid \mathbf{x})}\right]=\theta_{k}-\mathbf{x}^{\prime} \beta_{k} \quad k=0,1, \ldots, K-1$.

Additionally, the NPCRM can be fit via $K$ ordinary binary logistic regression models. Details and an example can be found in Hosmer and Lemeshow [1, p. 296].

\subsection{The partial proportional continuation ratio model}

If only some of the covariates violate the proportionality assumption in the CRM, then a more parsimonious model than the NPCRM should be considered. Under the partial proportional continuation ratio model (PPCRM) [25], two sets of regression coefficients are estimated: one set has proportional odds and the other set has non-proportional odds. For $k=0,1, \ldots, K-1$, this model has the form:

$$
r_{k}^{P P}(\mathbf{x})=\ln \left[\frac{P(Y=k \mid \mathbf{x})}{P(Y>k \mid \mathbf{x})}\right]=\theta_{k}-\mathbf{x}^{\prime} \beta-\mathbf{T}^{\prime} \gamma_{k} .
$$

Cole and Ananth [25] demonstrate a new method for fitting the PPCRM using a "person-threshold" data set. A separate binary logistic regression modelling approach is also valid because the PPCRM possesses the property of conditional independence.

\subsection{The adjacent category model}

The adjacent category model (ACM) proceeds by forming $K$ logits for all pairs of adjacent categories [9]. In this model, each response is compared to the next lower response. For $k=1,2, \ldots, K$, the ACM model is

$$
a_{k}(\mathbf{x})=\ln \left[\frac{P(Y=k \mid \mathbf{x})}{P(Y=k-1 \mid \mathbf{x})}\right]=\alpha_{k}+\mathbf{x}^{\prime} \beta .
$$

This model also assumes that the assumption of proportionality is satisfied by each variable in the model. The likelihood ratio test can be used to check the proportionality assumption in the adjacent category model in a global sense, but there is no valid approach to test if this assumption is satisfied individually.

The ACM is a constrained version of the MLRM. Thus, model parameters and standard error estimates can be directly obtained from the MLRM. The MLRM can be expressed in terms of the ACM as follows [1,9]:

(16)

$$
\begin{aligned}
\ln \left[\frac{P(Y=k \mid \mathbf{x})}{P(Y=0 \mid \mathbf{x})}\right] & =\ln \left[\frac{P(Y=1 \mid \mathbf{x})}{P(Y=0 \mid \mathbf{x})}\right]+\ln \left[\frac{P(Y=2 \mid \mathbf{x})}{P(Y=1 \mid \mathbf{x})}\right]+ \\
& \ldots+\ln \left[\frac{P(Y=k \mid \mathbf{x})}{P(Y=k-1 \mid \mathbf{x})}\right] \\
& =a_{1}(\mathbf{x})+a_{2}(\mathbf{x})+\ldots+a_{k}(\mathbf{x}) \\
& =\left(\alpha_{1}+\mathbf{x}^{\prime} \beta\right)+\left(\alpha_{2}+\mathbf{x}^{\prime} \beta\right)+ \\
& \ldots+\left(\alpha_{k}+\mathbf{x}^{\prime} \beta\right) \\
& =\left(\alpha_{1}+\alpha_{2}+\ldots+\alpha_{k}\right)+k \mathbf{x}^{\prime} \beta
\end{aligned}
$$

Thus, we see that the model in Equation (16) is a version of the MLRM in Equation (1) with intercept $\beta_{0 k}=\left(\alpha_{1}+\right.$ $\left.\alpha_{2}+\ldots+\alpha_{k}\right)$ and regression coefficient $\beta_{k}=k \beta$. In order to obtain the ACM estimates of $\alpha_{k}$ 's and $\beta$ 's from the MLRM, we fit a MLRM using the two constraints given below:

$$
\beta_{0 k}=\sum_{i=1}^{k} \alpha_{i} \quad \text { and } \quad \beta_{k}=k \beta
$$

This approach for fitting ACM via MLRM is explained for STATA software [30], and a real data example can be 
found in Hosmer and Lemeshow's book [1, p. 294]. Note that with this formalization, ACM gives rise to an ordinal interpretation in terms of adjacent odds where the MLRM does not.

The ACM became popular because of connections with the ordinal log-linear models [9]. The corresponding ordinal log-linear model for the adjacent category logit model is the linear-by-linear association model. For two-way tables, let the assigned row scores and column scores of ordinal variables be $u_{1}<u_{2}<\ldots<u_{I}$ and $v_{1}<v_{2}<\ldots<v_{I}$, respectively. Then, the linear-by-linear association model is

$$
\log \mu_{i j}=\lambda+\lambda_{i}^{X}+\lambda_{j}^{Y}+\beta u_{i} v_{j}
$$

with constraints such as $\lambda_{I}^{X}=\lambda_{J}^{Y}=0$. The term $\beta u_{i} v_{j}$ in Equation (18) represents the deviation of $\log \mu_{i j}$ from independence. The deviation is linear in the $Y$ scores at a fixed level of $X$ and linear in the $X$ scores at a fixed level of $Y$. Because of this property, this model is called the linearby-linear association model.

A logit formulation of the linear-by-linear association model treats $Y$ as a response and $X$ as explanatory. Using logits for adjacent response categories,

$$
\log \frac{\mu_{i, j+1}}{\mu_{i, j}}=\left(\lambda_{j+1}^{Y}-\lambda_{j}^{Y}\right)+\beta\left(v_{j+1}-v_{j}\right) u_{i},
$$

and for unit spaced $v_{j}$, the linear-by-linear association model simplifies to the ACM as

$$
\log \frac{\pi_{j+1 \mid i}}{\pi_{j \mid i}}=\alpha_{j}+\beta u_{i},
$$

where $\alpha_{j}=\lambda_{j+1}^{Y}-\lambda_{j}^{Y}$. In the linear-by-linear association model, local odds ratios for adjacent rows and columns have a common value $e^{\beta}$.

Additionally, using the connection between the ACM and the linear-by-linear association model, the parameter estimates in the ACM can be obtained from the Poisson loglikelihood. The log-likelihood in the linear-by-linear association model is

$$
\begin{aligned}
\mathbf{L}(\mu) & =n \lambda+\sum_{i} n_{i+} \lambda_{i}^{X}+\sum_{j} n_{+j} \lambda_{j}^{Y}+\beta \sum_{i} \sum_{j} u_{i} v_{j} n_{i j} \\
& -\sum_{i} \sum_{j} \exp \left(\lambda+\lambda_{i}^{X}+\lambda_{j}^{Y}+\beta u_{i} v_{j}\right) .
\end{aligned}
$$

Differentiating $\mathbf{L}(\mu)$ with respect to $\left(\lambda_{i}^{X}, \lambda_{j}^{Y}, \beta\right)$ and using iterative methods such as Newton-Raphson method yields the maximum likelihood fit.

\subsection{The non-proportional adjacent category model}

Like the proportional odds model and the continuation ratio model, the adjacent category logistic regression model assumes that the assumption of proportionality is satisfied by each variable in the model. The non-proportional adjacent category model (NPACM) relaxes the assumption of proportional odds for all variables in the model and is given as follows [31]:

$a_{k}^{N P}(\mathbf{x})=\ln \left[\frac{P(Y=k \mid \mathbf{x})}{P(Y=k-1 \mid \mathbf{x})}\right]=\alpha_{k}+\mathbf{x}^{\prime} \beta_{k} \quad k=1,2, \ldots, K$.

The NPACM is also a constrained version of the MLRM. Thus, model parameters and standard error estimates can be directly obtained from the MLRM as follows:

$$
\begin{aligned}
\ln \left[\frac{P(Y=k \mid \mathbf{x})}{P(Y=0 \mid \mathbf{x})}\right] & =\ln \left[\frac{P(Y=1 \mid \mathbf{x})}{P(Y=0 \mid \mathbf{x})}\right]+\ln \left[\frac{P(Y=2 \mid \mathbf{x})}{P(Y=1 \mid \mathbf{x})}\right]+ \\
& \ldots+\ln \left[\frac{P(Y=k \mid \mathbf{x})}{P(Y=k-1 \mid \mathbf{x})}\right] \\
& =a_{1}(\mathbf{x})+a_{2}(\mathbf{x})+\ldots+a_{k}(\mathbf{x}) \\
& =\left(\alpha_{1}+\mathbf{x}^{\prime} \beta_{1}\right)+\ldots+\left(\alpha_{k}+\mathbf{x}^{\prime} \beta_{k}\right) \\
& =\left(\alpha_{1}+\ldots+\alpha_{k}\right)+\mathbf{x}^{\prime}\left(\beta_{1}+\beta_{2}+\ldots+\beta_{k}\right) .
\end{aligned}
$$

Thus, we see that the model in Equation (22) is a version of the MLRM in Equation (1) with intercept $\beta_{0 k}=\left(\alpha_{1}+\right.$ $\left.\alpha_{2}+\ldots+\alpha_{k}\right)$ and regression coefficient $\beta_{k}=\left(\beta_{1}+\beta_{2}+\right.$ $\left.\ldots+\beta_{k}\right)$. In order to obtain the NPACM estimates of $\alpha_{k}$ 's and $\beta_{k}$ 's from the MLRM, we fit a MLRM using the two constraints given below:

$$
\beta_{0 k}=\sum_{i=1}^{k} \alpha_{i} \quad \text { and } \quad \beta_{k}=\sum_{i=1}^{k} \beta_{i} .
$$

Because the two constraints given in Equation (24) are additive rather than multiplicative, the NPACM and MLRM models have equal log-likelihoods. For this reason, the NPACM can be seen as an MLRM with a different parametrization (i.e., using adjacent logits rather than baseline logits).

\subsection{The partial proportional adjacent category model}

When some of the independent variables in the ACM do not meet the proportionality assumption, a need for a more parsimonious model than the NPACM naturally arises. Following the idea that was done before for the POM and the CRM, a partial proportional model for the ACM can be constructed. For $k=1,2, \ldots, K$ the partial proportional adjacent category model (PPACM) is

$$
a_{k}^{P P}(\mathbf{x})=\ln \left[\frac{P(Y=k \mid \mathbf{x})}{P(Y=k-1 \mid \mathbf{x})}\right]=\alpha_{k}+\mathbf{x}^{\prime} \beta+\mathbf{T}^{\prime} \gamma_{k} .
$$

Under the PPACM in Equation (25), two sets of regression coefficients should be estimated: one set (denoted by $\beta$ ) 
meets the proportional odds assumption, and a second set (denoted by $\gamma_{k}$ ) violates the proportional odds assumption.

At this point, the independent variables (and thus the corresponding coefficients) that meet or do not meet the proportionality assumption in the PPACM can be determined with the proposed Wald test statistic given in Section 5.

In order to estimate the $\beta$ and $\gamma_{k}$ 's in the PPACM, we can utilize the relationships between the adjacent category model, the non-proportional adjacent category model, and the multinomial logistic regression model which are given in Sections 3.7 and 3.8 .

Fitting (with the maximum likelihood estimation method) a multinomial logistic regression model using the two constraints given in Equation (24) gives the parameter estimates for a fully non-proportional adjacent category model (NPACM). On the other hand, fitting a multinomial logistic regression model using the two constraints given in Equation (17) gives the parameter estimates of a fully proportional adjacent category model (ACM). Then, fitting a multinomial logistic regression model using constraints given in Equation (17) for only some of the parameters in the model while leaving the rest of them without any constraints will give the parameter estimates of a partial version of the adjacent category model (PPACM). Yee [33] recently published the $\mathrm{R}$ codes (VGAM) for fitting the partial proportional adjacent category model.

\section{METHODS USED FOR CHECKING THE PROPORTIONAL ODDS ASSUMPTION}

\subsection{The likelihood ratio test}

Let ordinal dependent variable $Y$ take values of $k=$ $1,2, \ldots, K$ (note that, this display is different from that given as $k=0,1, \ldots, K$ previously), and the proportional odds model has $j$ independent variables $(j=1,2, \ldots, J)$. When the proportional odds assumption holds, the regression coefficients corresponding to the $j$ th independent variable $\left(\beta_{j k}\right)$ are equal to each other. This hypothesis can be formulated as follows [21]:

$$
H_{0}: \beta_{1 j}=\beta_{2 j}=\ldots=\beta_{(K-1) j} \quad j=1,2, \ldots, J .
$$

As the proportional odds model is nested within the nonproportional odds model, the likelihood ratio (LR) test, which evaluates differences in deviances, provides an omnibus test of proportionality. Under $H_{0}$, the LR test statistic is distributed as chi-square with degrees of freedom equal to the difference in the number of parameters between the nested models (which is $J(K-2)$ ) [25]. The LR test is a very useful tool, and it can be used to check the proportionality assumption in the continuation and adjacent category models in a global sense.

Another method, an approximate likelihood ratio test [32], is also an omnibus test of proportionality that tests whether the coefficients for all variables are simultaneously equal. However, it compares the log-likelihood of the proportional odds model to the log-likelihood obtained from pooling $K-1$ binary logistic regression models, adjusting for the correlation between $K-1$ binary logistic regression models. This approach is an approximation to the LR test because the log-likelihood obtained from pooling $K-1$ binary logistic regression models is only an approximation to the likelihood of the non-proportional odds model.

\subsection{Brant's Wald test}

The LR test is an omnibus test that does not show whether the proportionality assumption is violated for all independent variables or only for some. Brant [26] proposed a Wald test that tests the proportionality assumption for each variable individually and together in the proportional odds model. The approach is based on viewing the model in Equation (9) as a combination of $K-1$ correlated binary logistic regression regressions with outcomes defined by

$$
z_{k}= \begin{cases}1 & \text { if } Y>k \\ 0 & \text { if } Y \leq k\end{cases}
$$

Brant [26] proved that under the null hypothesis, the separate maximum likelihood estimates $\hat{\beta}_{k}^{*}=\left(\hat{\beta}_{1}^{\prime} \hat{\beta}_{2}^{\prime} \ldots \hat{\beta}_{K-1}^{\prime}\right)^{\prime}$ have a distribution that is asymptotically multivariate normal. Thus, the Wald test statistic, which is based on the correlated separate fits, provides an assessment of the proportionality assumption in the proportional odds model.

It is an important task to check the proportionality assumption in any ordinal logistic regression model. Bender and Grouven [2] showed that use of the POM can lead to invalid results if the main model assumption is violated, and they make a point for POM as follows:

\footnotetext{
"With increasing use of the POM, the misuse of it will also increase, especially due to application of this method to data with non-proportional odds. Unfortunately, the results of logistic regression models are poorly presented in medical research papers. Hence, an invalid application of the POM is seldom detectable for readers of medical journals."
}

Therefore, applying a proportionality test before proceeding with any of the ordinal logistic regression models is crucial. However, the existing methods for testing proportionality assumptions are restricted. An LR test is applicable for all of the ordinal logistic models, but it is only a way of testing proportionality in a global sense, and the Brant's test of Wald must be generalized for other ordinal logistic regression models such as the adjacent category model.

\section{AN ADAPTATION OF BRANT'S WALD TEST FOR THE ADJACENT CATEGORY MODEL}

Brant showed that the separate binary logistic regression estimates of $\hat{\beta}_{k}$ 's are asymptotically unbiased and follow a 
multivariate normal distribution [26]. Following the idea of Brant, we adapted a test for checking the proportionality assumption in the adjacent category model as follows:

1. The ACM uses adjacent category logits, so the first step of the proposed test statistic is to estimate $\hat{\beta}_{k}$ 's and $\operatorname{Var}\left(\hat{\beta_{k}}\right)$ 's from the $K-1$ binary logistic regressions with outcomes defined by

$$
z_{k}= \begin{cases}1 & \text { if } Y=k \\ 0 & \text { if } Y=k-1 \\ \text { Missing } & \text { else. }\end{cases}
$$

2. Estimate the probability that $z_{i k}=1$ given $x_{i}$ by using the below formula:

$$
\hat{\pi}_{k}\left(x_{i}\right)=\frac{e^{\hat{\tau}_{k}+\mathbf{x}^{\prime} \hat{\beta}_{k}}}{1+e^{\hat{\tau}_{k}+\mathbf{x}^{\prime} \hat{\beta}_{k}}} .
$$

Note that in this step, the probabilities $\left(\hat{\pi}_{k}\left(x_{i}\right)\right)$ that correspond to the missing categories of $z_{k}$ 's should remain as missing because there will not be any positive outcome probability that corresponds to the missing outcome category in the binary logistic regression model.

3. Estimate the covariance between $\hat{\beta}_{k}$ and $\hat{\beta}_{l}(k \leq l)$. Define:

$$
w_{i k l}=\hat{\pi}_{l}\left(x_{i}\right)-\hat{\pi}_{k}\left(x_{i}\right) \hat{\pi}_{l}\left(x_{i}\right)
$$

and let $W_{i k l}$ be a $N^{*} \times N^{*}$ diagonal matrix whose $i$ th element is $w_{i k l}$, and $N^{*}$ is the number of cases when we exclude the missing cases listwise. Let $\mathrm{X}$ be the $N^{*} \times(J+1)$ matrix with 1's in the first column and the independent variables in the remaining columns. Estimate the $\widehat{\operatorname{Var}}\left(\hat{\beta}_{k}, \hat{\beta}_{l}\right)$ by deleting the first row and column of

$$
\left(X^{\prime} W_{k k} X\right)^{-1}\left(X^{\prime} W_{k l} X\right)\left(X^{\prime} W_{l l} X\right)^{-1} .
$$

4. Combine all estimates. Define $\hat{\beta}_{k}^{*}=\left(\hat{\beta}_{1}^{\prime} \hat{\beta}_{2}^{\prime} \ldots \hat{\beta}_{K-1}^{\prime}\right)^{\prime}$ and

$$
\widehat{\operatorname{Var}}\left(\hat{\beta}_{k}^{*}\right)=\left(\begin{array}{ccc}
\widehat{\operatorname{Var}}\left(\hat{\beta}_{1}\right) & \ldots & \widehat{\operatorname{Var}}\left(\hat{\beta}_{1}, \hat{\beta}_{K-1}\right) \\
\vdots & \ddots & \vdots \\
\widehat{\operatorname{Var}}\left(\hat{\beta}_{K-1}, \hat{\beta}_{1}\right) & \ldots & \widehat{\operatorname{Var}}\left(\hat{\beta}_{1}\right)
\end{array}\right) .
$$

The diagonal elements $\widehat{\operatorname{Var}}\left(\hat{\beta}_{k}\right)$ are the covariance matrices from each binary regression. The off-diagonal elements were defined at step 3 .

5. Construct the Wald test of $H_{0}: \beta_{1}=\beta_{2}=\ldots=$ $\beta_{(K-1)}$. This hypothesis corresponds to $H_{0}: \mathbf{D} \beta^{*}=0$
Table 1. Frequencies in a $R \times C$ table

\begin{tabular}{ccccc}
\hline \hline Independent & \multicolumn{4}{c}{ Outcome Variable $\mathrm{Y}$} \\
\cline { 2 - 5 } Variable X & 1 & 2 & $\ldots$ & $\mathrm{C}$ \\
\hline 1 & $n_{11}$ & $n_{12}$ & $\ldots$ & $n_{1 C}$ \\
2 & $n_{21}$ & $n_{22}$ & $\ldots$ & $n_{2 C}$ \\
$\vdots$ & $\vdots$ & $\vdots$ & $\vdots$ & $\vdots$ \\
$\mathrm{R}$ & $n_{R 1}$ & $n_{R 2}$ & $\ldots$ & $n_{R C}$ \\
\hline
\end{tabular}

where

$$
\mathbf{D}=\left(\begin{array}{ccccc}
\mathbf{I} & -\mathbf{I} & \mathbf{0} & \ldots & \mathbf{0} \\
\mathbf{I} & \mathbf{0} & -\mathbf{I} & \ldots & \mathbf{0} \\
\vdots & \vdots & \vdots & \ddots & \vdots \\
\mathbf{I} & \mathbf{0} & \mathbf{0} & \ldots & -\mathbf{I}
\end{array}\right)
$$

I is a $(J+1) \times(J+1)$ identity matrix, and $\mathbf{0}$ is a $(J+1) \times(J+1)$ matrix of 0 's. The Wald test statistic

$$
W=\left(\mathbf{D} \hat{\beta}^{*}\right)^{\prime}\left[\mathbf{D} \widehat{\operatorname{Var}}\left(\hat{\beta}^{*}\right) \mathbf{D}^{\prime}\right]^{-1}\left(\mathbf{D} \hat{\beta}^{*}\right)
$$

distributes as chi-square with $J(K-2)$ degrees of freedom.

6. Construct tests for individual variables. The hypothesis $H_{0}: \beta_{1 j}=\beta_{2 j}=\ldots=\beta_{(K-1) j}$ can be tested by selecting only those rows and columns of $\mathbf{D}, \hat{\beta}^{*}$ and $\widehat{\operatorname{Var}}\left(\hat{\beta}^{*}\right)$ that correspond to the coefficients being tested. The resulting test statistic has $K-2$ degrees of freedom. For the overall and individual tests, if the Wald test statistic is big enough, the proportionality assumption can be rejected.

\section{MONTE CARLO SIMULATION}

In Section 6.1, the distributional properties of the proposed test in Equation (34) are studied under $H_{0}$ by using a Monte Carlo simulation. The appropriateness of chi-square approximation is examined using some graphical methods such as Q-Q plots, probability distribution and cumulative distribution plots. Then, type I error rates of the proposed test and LR test are compared under the same scenarios. In Section 6.2, another Monte Carlo simulation study is conducted to compare the proposed test and LR test in terms of power under alternative hypotheses.

\subsection{Simulation of the adapted test statistic under $\boldsymbol{H}_{0}$}

Suppose that the ordinal dependent variable has $C$ levels $(j=1,2, \ldots, C)$ and the independent variable has $R$ levels $(i=1,2, \ldots, R)$, and let the frequencies in the $R \times C$ table be given as Table 1 .

In the $R \times C$ table, the null hypothesis of proportionality or in other words, the common odds ratio across the adjacent 
categories, can be expressed as

$$
H_{0}: \frac{n_{i j} \cdot n_{(i+1)(j+1)}}{n_{i(j+1)} \cdot n_{(i+1) j}}=O R
$$

for $i=1,2, \ldots, R$ and $j=1,2, \ldots, C$. To generate frequencies from the multinomial distribution under Equation (35), one must calculate the probabilities of the adjacent categories that gives the same odds ratio across every category. This calculation is simple for a $2 \times 3$ table; however, for an $R \times C$ table, it can be tedious. Therefore, upon generating frequencies in an $R \times C$ table under $H_{0}$, we use the connection between the adjacent category model and the linear-by-linear model that is given in Section 3.7.

Data for the first simulation study is generated under the following linear-by-linear model:

$$
\log \mu_{i j}=\lambda+\lambda_{i}^{X}+\lambda_{j}^{Y}+\beta u_{i} v_{j}
$$

where $\lambda$ is the general effect parameter, and $\lambda_{i}^{X}$ and $\lambda_{j}^{Y}$ are the main effects for variables $\mathrm{X}$ and $\mathrm{Y}$, with constraints $\lambda_{I}^{X}=\lambda_{J}^{Y}=0$. Under the linear-by-linear model, we guaranteed that the generated frequencies in the $R \times C$ table will satisfy Equation (35) and the common adjacent odds ratio as $\exp (\beta)=O R$.

In large samples, the parameters $\left(\lambda, \lambda_{i}^{X}\right.$ and $\left.\lambda_{j}^{Y}\right)$ in the linear-by-linear model are approximately normally distributed [9], and the formal significance testing of the parameters can be performed using a Wald test statistic. Therefore, we used the normal distribution for generating the parameters of the linear-by-linear model. Mean values of $\beta$ were taken as $\beta=0,0.182,0.262$ to obtain the common odds ratio values of $O R=1,1.2,1.3$. We did not consider using any $\mathrm{OR}$ values greater than 1.3 , because when we generate cell frequencies under $H_{0}$, for let's say $\mathrm{OR}=1.5$ in the $4 \times 5$ table, six cells $(6 / 20=30 \%$ of the cells $)$ have expected value less than five in the table, even if $N=1,000$. Additionally when we increase the OR, for example when $\mathrm{OR}=2$, fourteen cells $(14 / 20=70 \%$ of the cells $)$ have expected value less than five, which may lead us to invalid results for the simulation study.

The sample size was taken as $N=300,500,1,000$, and we applied the ad hoc adjustment procedure of adding 0.50 to each cell if the generated $R \times C$ table contained cells with 0 frequencies. Mean, standard deviation of the linearby-linear model parameters, common adjacent odds ratios, $R$ and $C$ values, and sample sizes that were used in the first simulation study are given in Table 2 .

$R \times C$ tables were generated 1,000 times from the given $R$, $C, \beta, \lambda, \lambda_{i}^{X}$ and $\lambda_{j}^{Y}$ combination, and in each run, the proposed Wald test statistic, which is given in Equation (34), was computed. We also computed the proportionality tests for the individual variables in the model. The appropriateness of the chi-square approximation of the omnibus and the individual test statistics were investigated using graphical methods such as the probability density function, cumulative distribution function and Q-Q plots. We also calculated the likelihood ratio test statistics using the log-likelihood of the non-proportional adjacent category model $\left(L_{N P A C M}\right)$ and the adjacent category model $\left(L_{A C M}\right)$, and we compared the proposed test statistic with the likelihood ratio test results. We obtained the estimated type I error rates for the proposed Wald and the LR tests as the percentage of times the test rejects the null hypothesis when the null hypothesis is true.

\subsection{Power comparison of the adapted test statistic and the LR test}

Data for the second simulation study is generated under the following log-linear model:

$$
\log \mu_{i j}=\lambda+\lambda_{i}^{X}+\lambda_{j}^{Y}+\beta_{i j} u_{i} v_{j}
$$

where $\lambda$ is the general effect parameter, and $\lambda_{i}^{X}$ and $\lambda_{j}^{Y}$ are the main effects for variables $\mathrm{X}$ and $\mathrm{Y}$, with constraints $\lambda_{I}^{X}=\lambda_{J}^{Y}=0$. For $i=1,2, \ldots, R$ and $j=1,2, \ldots, C, \beta_{i j}$ 's denote different log-adjacent odds ratios across categories. The values of $\beta_{i j}$ are generated stochastically from the normal distribution with different mean and standard deviation. In order to control the degree of non-proportionality of the log-adjacent odds ratios, we used their standard deviations, such that when the standard deviation of $\beta_{i j}$ is increased, the degree of non-proportionality increases. Mean, standard deviation of the linear-by-linear model parameters, log-adjacent odds ratios $\left(\beta_{i j}\right), R$ and $C$ values, and sample sizes that were used in the second simulation study are given in Table 3 .

$R \times C$ tables were generated from the given $R, C, \beta_{i j}, \lambda$, $\lambda_{i}^{X}$ and $\lambda_{j}^{Y}$ combinations. $\beta_{i j}$ 's are generated under the normal distribution with parameters $N(0,0.02), N(0.182,0.03)$, and $N(0.262,0.04)$ for small effect sizes, and $N(0,0.2)$, $N(0.182,0.3)$, and $N(0.262,0.4)$ for greater effect sizes. In each run, the proposed Wald and the likelihood ratio test statistics were calculated. We obtained the estimated power of tests as the percentage of times the test rejects the null hypothesis when the null hypothesis is false. The simulation codes were developed in $\mathrm{R}$ (Version 3.0.2) and the results are summarized in the next section.

\section{RESULTS OF THE SIMULATION STUDY}

The results of the first simulation study are given in Table 4 .

In Table 4, the mean values of the omnibus LR and the proposed test statistics that were computed from 1,000 random samples are given in columns 3 and 4 . The proposed Wald test statistic and the LR test statistic should be distributed as chi-square with a mean of $J(K-2)$ (note that, for a $R \times C$ table, the number of independent variables is $J=R-1$ and $K=C$ ) degrees of freedom. As seen in these columns, the mean values of the omnibus version of the pro- 
Table 2. Mean, standard deviation of the linear-by-linear model parameters, common adjacent odds ratios, $R \times C$ values, and sample size combinations that are used under $H_{0}$

\begin{tabular}{|c|c|c|c|c|c|c|}
\hline \multirow{2}{*}{$\begin{array}{l}\text { Scenario } \\
\text { Number }\end{array}$} & \multicolumn{3}{|c|}{ Distribution of the parameters } & \multirow{2}{*}{$\begin{array}{c}\text { Common } \\
\text { Adjacent } O R\end{array}$} & \multirow[b]{2}{*}{$R \times C$} & \multirow{2}{*}{$\begin{array}{c}\text { Total } \\
\text { Sample Size }\end{array}$} \\
\hline & $\lambda$ & $\lambda_{i}^{X}$ & $\lambda_{j}^{Y}$ & & & \\
\hline 1 & $\mathrm{~N}(1,1)$ & $\mathrm{N}(0,1)$ & $\mathrm{N}(0,1)$ & $O R=1$ & $3 \times 4$ & 300 \\
\hline 2 & $\mathrm{~N}(1,1)$ & $\mathrm{N}(0,1)$ & $\mathrm{N}(0,1)$ & $O R=1$ & $3 \times 4$ & 500 \\
\hline 3 & $\mathrm{~N}(1,1)$ & $\mathrm{N}(0,1)$ & $\mathrm{N}(0,1)$ & $O R=1$ & $4 \times 5$ & 300 \\
\hline 4 & $\mathrm{~N}(1,1)$ & $\mathrm{N}(0,1)$ & $\mathrm{N}(0,1)$ & $O R=1$ & $4 \times 5$ & 500 \\
\hline 5 & $\mathrm{~N}(1,1)$ & $\mathrm{N}(0,1)$ & $\mathrm{N}(0,1)$ & $O R=1.2$ & $3 \times 4$ & 500 \\
\hline 6 & $\mathrm{~N}(1,1)$ & $\mathrm{N}(0,1)$ & $\mathrm{N}(0,1)$ & $O R=1.2$ & $3 \times 4$ & 1000 \\
\hline 7 & $\mathrm{~N}(1,1)$ & $\mathrm{N}(0,1)$ & $\mathrm{N}(0,1)$ & $O R=1.2$ & $4 \times 5$ & 500 \\
\hline 8 & $\mathrm{~N}(1,1)$ & $\mathrm{N}(0,1)$ & $\mathrm{N}(0,1)$ & $O R=1.2$ & $4 \times 5$ & 1000 \\
\hline 9 & $\mathrm{~N}(1,1)$ & $\mathrm{N}(0,1)$ & $\mathrm{N}(0,1)$ & $O R=1.3$ & $3 \times 4$ & 500 \\
\hline 10 & $\mathrm{~N}(1,1)$ & $\mathrm{N}(0,1)$ & $\mathrm{N}(0,1)$ & $O R=1.3$ & $3 \times 4$ & 1000 \\
\hline 11 & $\mathrm{~N}(1,1)$ & $\mathrm{N}(0,1)$ & $\mathrm{N}(0,1)$ & $O R=1.3$ & $4 \times 5$ & 500 \\
\hline 12 & $\mathrm{~N}(1,1)$ & $\mathrm{N}(0,1)$ & $\mathrm{N}(0,1)$ & $O R=1.3$ & $4 \times 5$ & 1000 \\
\hline 13 & $\mathrm{~N}(1,1)$ & $\mathrm{N}(1,0.5)$ & $\mathrm{N}(1,0.5)$ & $O R=1$ & $3 \times 4$ & 300 \\
\hline 14 & $\mathrm{~N}(1,1)$ & $\mathrm{N}(1,0.5)$ & $\mathrm{N}(1,0.5)$ & $O R=1$ & $3 \times 4$ & 500 \\
\hline 15 & $\mathrm{~N}(1,1)$ & $\mathrm{N}(1,0.5)$ & $\mathrm{N}(1,0.5)$ & $O R=1$ & $4 \times 5$ & 300 \\
\hline 16 & $\mathrm{~N}(1,1)$ & $\mathrm{N}(1,0.5)$ & $\mathrm{N}(1,0.5)$ & $O R=1$ & $4 \times 5$ & 500 \\
\hline 17 & $\mathrm{~N}(1,1)$ & $\mathrm{N}(1,0.5)$ & $\mathrm{N}(1,0.5)$ & $O R=1.2$ & $3 \times 4$ & 500 \\
\hline 18 & $\mathrm{~N}(1,1)$ & $\mathrm{N}(1,0.5)$ & $\mathrm{N}(1,0.5)$ & $O R=1.2$ & $3 \times 4$ & 1000 \\
\hline 19 & $\mathrm{~N}(1,1)$ & $\mathrm{N}(1,0.5)$ & $\mathrm{N}(1,0.5)$ & $O R=1.2$ & $4 \times 5$ & 500 \\
\hline 20 & $\mathrm{~N}(1,1)$ & $\mathrm{N}(1,0.5)$ & $\mathrm{N}(1,0.5)$ & $O R=1.2$ & $4 \times 5$ & 1000 \\
\hline 21 & $\mathrm{~N}(1,1)$ & $\mathrm{N}(1,0.5)$ & $\mathrm{N}(1,0.5)$ & $O R=1.3$ & $3 \times 4$ & 500 \\
\hline 22 & $\mathrm{~N}(1,1)$ & $\mathrm{N}(1,0.5)$ & $\mathrm{N}(1,0.5)$ & $O R=1.3$ & $3 \times 4$ & 1000 \\
\hline 23 & $\mathrm{~N}(1,1)$ & $\mathrm{N}(1,0.5)$ & $\mathrm{N}(1,0.5)$ & $O R=1.3$ & $4 \times 5$ & 500 \\
\hline 24 & $\mathrm{~N}(1,1)$ & $\mathrm{N}(1,0.5)$ & $\mathrm{N}(1,0.5)$ & $O R=1.3$ & $4 \times 5$ & 1000 \\
\hline 25 & $\mathrm{~N}(1,1)$ & $\mathrm{N}(1,1)$ & $\mathrm{N}(1,1)$ & $O R=1$ & $3 \times 4$ & 300 \\
\hline 26 & $\mathrm{~N}(1,1)$ & $\mathrm{N}(1,1)$ & $\mathrm{N}(1,1)$ & $O R=1$ & $3 \times 4$ & 500 \\
\hline 27 & $\mathrm{~N}(1,1)$ & $\mathrm{N}(1,1)$ & $\mathrm{N}(1,1)$ & $O R=1$ & $4 \times 5$ & 300 \\
\hline 28 & $\mathrm{~N}(1,1)$ & $\mathrm{N}(1,1)$ & $\mathrm{N}(1,1)$ & $O R=1$ & $4 \times 5$ & 500 \\
\hline 29 & $\mathrm{~N}(1,1)$ & $\mathrm{N}(1,1)$ & $\mathrm{N}(1,1)$ & $O R=1.2$ & $3 \times 4$ & 500 \\
\hline 30 & $\mathrm{~N}(1,1)$ & $\mathrm{N}(1,1)$ & $\mathrm{N}(1,1)$ & $O R=1.2$ & $3 \times 4$ & 1000 \\
\hline 31 & $\mathrm{~N}(1,1)$ & $\mathrm{N}(1,1)$ & $\mathrm{N}(1,1)$ & $O R=1.2$ & $4 \times 5$ & 500 \\
\hline 32 & $\mathrm{~N}(1,1)$ & $\mathrm{N}(1,1)$ & $\mathrm{N}(1,1)$ & $O R=1.2$ & $4 \times 5$ & 1000 \\
\hline 33 & $\mathrm{~N}(1,1)$ & $\mathrm{N}(1,1)$ & $\mathrm{N}(1,1)$ & $O R=1.3$ & $3 \times 4$ & 500 \\
\hline 34 & $\mathrm{~N}(1,1)$ & $\mathrm{N}(1,1)$ & $\mathrm{N}(1,1)$ & $O R=1.3$ & $3 \times 4$ & 1000 \\
\hline 35 & $\mathrm{~N}(1,1)$ & $\mathrm{N}(1,1)$ & $\mathrm{N}(1,1)$ & $O R=1.3$ & $4 \times 5$ & 500 \\
\hline 36 & $\mathrm{~N}(1,1)$ & $\mathrm{N}(1,1)$ & $\mathrm{N}(1,1)$ & $O R=1.3$ & $4 \times 5$ & 1000 \\
\hline
\end{tabular}

posed test statistic are approximately $J(K-2)$. Again, the mean values of the omnibus version of the proposed test statistic are smaller than the likelihood ratio test statistics.

The fifth and sixth columns of Table 4 give the percentage of rejections of the proposed test statistic and the LR test statistic under the $\chi_{J(K-2)}^{2}$ distribution. As seen in these columns, type I error rates of both test statistics are around the 0.05 level, while the proposed test tends to be more conservative than the likelihood ratio test statistic.

The appropriateness of the chi-square approximation of the omnibus version of the proposed test statistic was investigated using pdf, cdf and Q-Q plots. All of the graphs are not given here to save space; only the graphical results related to scenario number 18 and 20 are given in Figure 1 and Figure 2, respectively.
In Figure 1, the solid lines represent the $\chi_{4}^{2}$ distribution (note that for the $18^{t h}$ scenario the degrees of freedom is equal to 4) and the dotted lines represent the omnibus version of the proposed Wald test statistic (in Figure 1 part (a)) and LR test statistic values (in Figure 1 part (b)) which are calculated from 1,000 replications. As seen from that figure, the solid and dotted lines overlap. Also in the Q-Q plot, the values of the omnibus version of the proposed Wald test statistic lie on the $45^{\circ}$ line.

In Figure 2, the solid lines represent the $\chi_{9}^{2}$ distribution (note that for the $20^{t h}$ scenario the degrees of freedom is equal to 9) and the dotted lines represent the omnibus version of the proposed Wald test statistic (in Figure 2 part (a)) and LR test statistic values (in Figure 2 part (b)) which are calculated from 1,000 replications. As seen from that fig- 
Table 3. Mean, standard deviation of the linear-by-linear model parameters, log-adjacent odds ratios, $R \times C$ values, and sample size combinations that are used under the alternative hypothesis

\begin{tabular}{|c|c|c|c|c|c|c|}
\hline \multirow{2}{*}{$\begin{array}{l}\text { Scenario } \\
\text { Number }\end{array}$} & \multicolumn{4}{|c|}{ Distribution of the parameters } & \multirow[b]{2}{*}{$R \times C$} & \multirow{2}{*}{$\begin{array}{c}\text { Total } \\
\text { Sample Size }\end{array}$} \\
\hline & $\lambda$ & $\lambda_{i}^{X}$ & $\lambda_{j}^{Y}$ & $\beta_{i j}$ & & \\
\hline 1 & $\mathrm{~N}(1,1)$ & $\mathrm{N}(0,1)$ & $\mathrm{N}(0,1)$ & $\mathrm{N}(0,0.02)$ & $3 \times 4$ & 300 \\
\hline 2 & $\mathrm{~N}(1,1)$ & $\mathrm{N}(0,1)$ & $\mathrm{N}(0,1)$ & $\mathrm{N}(0,0.2)$ & $3 \times 4$ & 300 \\
\hline 3 & $\mathrm{~N}(1,1)$ & $\mathrm{N}(0,1)$ & $\mathrm{N}(0,1)$ & $\mathrm{N}(0,0.02)$ & $3 \times 4$ & 500 \\
\hline 4 & $\mathrm{~N}(1,1)$ & $\mathrm{N}(0,1)$ & $\mathrm{N}(0,1)$ & $\mathrm{N}(0,0.2)$ & $3 \times 4$ & 500 \\
\hline 5 & $\mathrm{~N}(1,1)$ & $\mathrm{N}(0,1)$ & $\mathrm{N}(0,1)$ & $\mathrm{N}(0,0.02)$ & $4 \times 5$ & 300 \\
\hline 6 & $\mathrm{~N}(1,1)$ & $\mathrm{N}(0,1)$ & $\mathrm{N}(0,1)$ & $\mathrm{N}(0,0.2)$ & $4 \times 5$ & 300 \\
\hline 7 & $\mathrm{~N}(1,1)$ & $\mathrm{N}(0,1)$ & $\mathrm{N}(0,1)$ & $\mathrm{N}(0,0.02)$ & $4 \times 5$ & 500 \\
\hline 8 & $\mathrm{~N}(1,1)$ & $\mathrm{N}(0,1)$ & $\mathrm{N}(0,1)$ & $\mathrm{N}(0,0.2)$ & $4 \times 5$ & 500 \\
\hline 9 & $\mathrm{~N}(1,1)$ & $\mathrm{N}(0,1)$ & $\mathrm{N}(0,1)$ & $\mathrm{N}(0.182,0.03)$ & $3 \times 4$ & 500 \\
\hline 10 & $\mathrm{~N}(1,1)$ & $\mathrm{N}(0,1)$ & $\mathrm{N}(0,1)$ & $\mathrm{N}(0.182,0.3)$ & $3 \times 4$ & 500 \\
\hline 11 & $\mathrm{~N}(1,1)$ & $\mathrm{N}(0,1)$ & $\mathrm{N}(0,1)$ & $\mathrm{N}(0.182,0.03)$ & $3 \times 4$ & 1000 \\
\hline 12 & $\mathrm{~N}(1,1)$ & $\mathrm{N}(0,1)$ & $\mathrm{N}(0,1)$ & $\mathrm{N}(0.182,0.3)$ & $3 \times 4$ & 1000 \\
\hline 13 & $\mathrm{~N}(1,1)$ & $\mathrm{N}(0,1)$ & $\mathrm{N}(0,1)$ & $\mathrm{N}(0.182,0.03)$ & $4 \times 5$ & 500 \\
\hline 14 & $\mathrm{~N}(1,1)$ & $\mathrm{N}(0,1)$ & $\mathrm{N}(0,1)$ & $\mathrm{N}(0.182,0.3)$ & $4 \times 5$ & 500 \\
\hline 15 & $\mathrm{~N}(1,1)$ & $\mathrm{N}(0,1)$ & $\mathrm{N}(0,1)$ & $\mathrm{N}(0.182,0.03)$ & $4 \times 5$ & 1000 \\
\hline 16 & $\mathrm{~N}(1,1)$ & $\mathrm{N}(0,1)$ & $\mathrm{N}(0,1)$ & $\mathrm{N}(0.182,0.3)$ & $4 \times 5$ & 1000 \\
\hline 17 & $\mathrm{~N}(1,1)$ & $\mathrm{N}(0,1)$ & $\mathrm{N}(0,1)$ & $\mathrm{N}(0.262,0.04)$ & $3 \times 4$ & 500 \\
\hline 18 & $\mathrm{~N}(1,1)$ & $\mathrm{N}(0,1)$ & $\mathrm{N}(0,1)$ & $\mathrm{N}(0.262,0.4)$ & $3 \times 4$ & 500 \\
\hline 19 & $\mathrm{~N}(1,1)$ & $\mathrm{N}(0,1)$ & $\mathrm{N}(0,1)$ & $\mathrm{N}(0.262,0.04)$ & $3 \times 4$ & 1000 \\
\hline 20 & $\mathrm{~N}(1,1)$ & $\mathrm{N}(0,1)$ & $\mathrm{N}(0,1)$ & $\mathrm{N}(0.262,0.4)$ & $3 \times 4$ & 1000 \\
\hline 21 & $\mathrm{~N}(1,1)$ & $\mathrm{N}(0,1)$ & $\mathrm{N}(0,1)$ & $\mathrm{N}(0.262,0.04)$ & $4 \times 5$ & 500 \\
\hline 22 & $\mathrm{~N}(1,1)$ & $\mathrm{N}(0,1)$ & $\mathrm{N}(0,1)$ & $\mathrm{N}(0.262,0.4)$ & $4 \times 5$ & 500 \\
\hline 23 & $\mathrm{~N}(1,1)$ & $\mathrm{N}(0,1)$ & $\mathrm{N}(0,1)$ & $\mathrm{N}(0.262,0.04)$ & $4 \times 5$ & 1000 \\
\hline 24 & $\mathrm{~N}(1,1)$ & $\mathrm{N}(0,1)$ & $\mathrm{N}(0,1)$ & $\mathrm{N}(0.262,0.4)$ & $4 \times 5$ & 1000 \\
\hline 25 & $\mathrm{~N}(1,1)$ & $\mathrm{N}(1,0.5)$ & $\mathrm{N}(1,0.5)$ & $\mathrm{N}(0,0.02)$ & $3 \times 4$ & 300 \\
\hline 26 & $\mathrm{~N}(1,1)$ & $\mathrm{N}(1,0.5)$ & $\mathrm{N}(1,0.5)$ & $\mathrm{N}(0,0.2)$ & $3 \times 4$ & 300 \\
\hline 27 & $\mathrm{~N}(1,1)$ & $\mathrm{N}(1,0.5)$ & $\mathrm{N}(1,0.5)$ & $\mathrm{N}(0,0.02)$ & $3 \times 4$ & 500 \\
\hline 28 & $\mathrm{~N}(1,1)$ & $\mathrm{N}(1,0.5)$ & $\mathrm{N}(1,0.5)$ & $\mathrm{N}(0,0.2)$ & $3 \times 4$ & 500 \\
\hline 29 & $\mathrm{~N}(1,1)$ & $\mathrm{N}(1,0.5)$ & $\mathrm{N}(1,0.5)$ & $\mathrm{N}(0,0.02)$ & $4 \times 5$ & 300 \\
\hline 30 & $\mathrm{~N}(1,1)$ & $\mathrm{N}(1,0.5)$ & $\mathrm{N}(1,0.5)$ & $\mathrm{N}(0,0.2)$ & $4 \times 5$ & 300 \\
\hline 31 & $\mathrm{~N}(1,1)$ & $\mathrm{N}(1,0.5)$ & $\mathrm{N}(1,0.5)$ & $\mathrm{N}(0,0.02)$ & $4 \times 5$ & 500 \\
\hline 32 & $\mathrm{~N}(1,1)$ & $\mathrm{N}(1,0.5)$ & $\mathrm{N}(1,0.5)$ & $\mathrm{N}(0,0.2)$ & $4 \times 5$ & 500 \\
\hline 33 & $\mathrm{~N}(1,1)$ & $\mathrm{N}(1,0.5)$ & $\mathrm{N}(1,0.5)$ & $\mathrm{N}(0.182,0.03)$ & $3 \times 4$ & 500 \\
\hline 34 & $\mathrm{~N}(1,1)$ & $\mathrm{N}(1,0.5)$ & $\mathrm{N}(1,0.5)$ & $\mathrm{N}(0.182,0.3)$ & $3 \times 4$ & 500 \\
\hline 35 & $\mathrm{~N}(1,1)$ & $\mathrm{N}(1,0.5)$ & $\mathrm{N}(1,0.5)$ & $\mathrm{N}(0.182,0.03)$ & $3 \times 4$ & 1000 \\
\hline 36 & $\mathrm{~N}(1,1)$ & $\mathrm{N}(1,0.5)$ & $\mathrm{N}(1,0.5)$ & $\mathrm{N}(0.182,0.3)$ & $3 \times 4$ & 1000 \\
\hline 37 & $\mathrm{~N}(1,1)$ & $\mathrm{N}(1,0.5)$ & $\mathrm{N}(1,0.5)$ & $\mathrm{N}(0.182,0.03)$ & $4 \times 5$ & 500 \\
\hline 38 & $\mathrm{~N}(1,1)$ & $\mathrm{N}(1,0.5)$ & $\mathrm{N}(1,0.5)$ & $\mathrm{N}(0.182,0.3)$ & $4 \times 5$ & 500 \\
\hline 39 & $\mathrm{~N}(1,1)$ & $\mathrm{N}(1,0.5)$ & $\mathrm{N}(1,0.5)$ & $\mathrm{N}(0.182,0.03)$ & $4 \times 5$ & 1000 \\
\hline 40 & $\mathrm{~N}(1,1)$ & $\mathrm{N}(1,0.5)$ & $\mathrm{N}(1,0.5)$ & $\mathrm{N}(0.182,0.3)$ & $4 \times 5$ & 1000 \\
\hline 41 & $\mathrm{~N}(1,1)$ & $\mathrm{N}(1,0.5)$ & $\mathrm{N}(1,0.5)$ & $\mathrm{N}(0.262,0.04)$ & $3 \times 4$ & 500 \\
\hline 42 & $\mathrm{~N}(1,1)$ & $\mathrm{N}(1,0.5)$ & $\mathrm{N}(1,0.5)$ & $\mathrm{N}(0.262,0.4)$ & $3 \times 4$ & 500 \\
\hline 43 & $\mathrm{~N}(1,1)$ & $\mathrm{N}(1,0.5)$ & $\mathrm{N}(1,0.5)$ & $\mathrm{N}(0.262,0.04)$ & $3 \times 4$ & 1000 \\
\hline 44 & $\mathrm{~N}(1,1)$ & $\mathrm{N}(1,0.5)$ & $\mathrm{N}(1,0.5)$ & $\mathrm{N}(0.262,0.4)$ & $3 \times 4$ & 1000 \\
\hline 45 & $\mathrm{~N}(1,1)$ & $\mathrm{N}(1,0.5)$ & $\mathrm{N}(1,0.5)$ & $\mathrm{N}(0.262,0.04)$ & $4 \times 5$ & 500 \\
\hline 46 & $\mathrm{~N}(1,1)$ & $\mathrm{N}(1,0.5)$ & $\mathrm{N}(1,0.5)$ & $\mathrm{N}(0.262,0.4)$ & $4 \times 5$ & 500 \\
\hline 47 & $\mathrm{~N}(1,1)$ & $\mathrm{N}(1,0.5)$ & $\mathrm{N}(1,0.5)$ & $\mathrm{N}(0.262,0.04)$ & $4 \times 5$ & 1000 \\
\hline 48 & $\mathrm{~N}(1,1)$ & $\mathrm{N}(1,0.5)$ & $\mathrm{N}(1,0.5)$ & $\mathrm{N}(0.262,0.4)$ & $4 \times 5$ & 1000 \\
\hline
\end{tabular}

ure, the solid and dotted lines overlap. In the Q-Q plot, the the omnibus version of the proposed Wald test statistic has values of the omnibus version of the proposed Wald test a chi-square distribution under the $H_{0}$ for the $R \times C$ table. statistic also lie on the $45^{\circ}$ line.

In Table 4 , the mean values of the individual version of

Therefore, after examining the results, we concluded that the proposed test statistic that were computed from 1,000 
Table 3. (Continued)

\begin{tabular}{|c|c|c|c|c|c|c|}
\hline \multirow{2}{*}{$\begin{array}{l}\text { Scenario } \\
\text { Number }\end{array}$} & \multicolumn{4}{|c|}{ Distribution of the parameters } & \multirow[b]{2}{*}{$R \times C$} & \multirow{2}{*}{$\begin{array}{c}\text { Total } \\
\text { Sample Siz }\end{array}$} \\
\hline & $\lambda$ & $\lambda_{i}^{X}$ & $\lambda_{j}^{Y}$ & $\beta_{i j}$ & & \\
\hline 49 & $\mathrm{~N}(1,1)$ & $\mathrm{N}(1,1)$ & $\mathrm{N}(1,1)$ & $\mathrm{N}(0,0.02)$ & $3 \times 4$ & 300 \\
\hline 50 & $\mathrm{~N}(1,1)$ & $\mathrm{N}(1,1)$ & $\mathrm{N}(1,1)$ & $\mathrm{N}(0,0.2)$ & $3 \times 4$ & 300 \\
\hline 51 & $\mathrm{~N}(1,1)$ & $\mathrm{N}(1,1)$ & $\mathrm{N}(1,1)$ & $\mathrm{N}(0,0.02)$ & $3 \times 4$ & 500 \\
\hline 52 & $\mathrm{~N}(1,1)$ & $\mathrm{N}(1,1)$ & $\mathrm{N}(1,1)$ & $\mathrm{N}(0,0.2)$ & $3 \times 4$ & 500 \\
\hline 53 & $\mathrm{~N}(1,1)$ & $\mathrm{N}(1,1)$ & $\mathrm{N}(1,1)$ & $\mathrm{N}(0,0.02)$ & $4 \times 5$ & 300 \\
\hline 54 & $\mathrm{~N}(1,1)$ & $\mathrm{N}(1,1)$ & $\mathrm{N}(1,1)$ & $\mathrm{N}(0,0.2)$ & $4 \times 5$ & 300 \\
\hline 55 & $\mathrm{~N}(1,1)$ & $\mathrm{N}(1,1)$ & $\mathrm{N}(1,1)$ & $\mathrm{N}(0,0.02)$ & $4 \times 5$ & 500 \\
\hline 56 & $\mathrm{~N}(1,1)$ & $\mathrm{N}(1,1)$ & $\mathrm{N}(1,1)$ & $\mathrm{N}(0,0.2)$ & $4 \times 5$ & 500 \\
\hline 57 & $\mathrm{~N}(1,1)$ & $\mathrm{N}(1,1)$ & $\mathrm{N}(1,1)$ & $\mathrm{N}(0.182,0.03)$ & $3 \times 4$ & 500 \\
\hline 58 & $\mathrm{~N}(1,1)$ & $\mathrm{N}(1,1)$ & $\mathrm{N}(1,1)$ & $\mathrm{N}(0.182,0.3)$ & $3 \times 4$ & 500 \\
\hline 59 & $\mathrm{~N}(1,1)$ & $\mathrm{N}(1,1)$ & $\mathrm{N}(1,1)$ & $\mathrm{N}(0.182,0.03)$ & $3 \times 4$ & 1000 \\
\hline 60 & $\mathrm{~N}(1,1)$ & $\mathrm{N}(1,1)$ & $\mathrm{N}(1,1)$ & $\mathrm{N}(0.182,0.3)$ & $3 \times 4$ & 1000 \\
\hline 61 & $\mathrm{~N}(1,1)$ & $\mathrm{N}(1,1)$ & $\mathrm{N}(1,1)$ & $\mathrm{N}(0.182,0.03)$ & $4 \times 5$ & 500 \\
\hline 62 & $\mathrm{~N}(1,1)$ & $\mathrm{N}(1,1)$ & $\mathrm{N}(1,1)$ & $\mathrm{N}(0.182,0.3)$ & $4 \times 5$ & 500 \\
\hline 63 & $\mathrm{~N}(1,1)$ & $\mathrm{N}(1,1)$ & $\mathrm{N}(1,1)$ & $\mathrm{N}(0.182,0.03)$ & $4 \times 5$ & 1000 \\
\hline 64 & $\mathrm{~N}(1,1)$ & $\mathrm{N}(1,1)$ & $\mathrm{N}(1,1)$ & $\mathrm{N}(0.182,0.3)$ & $4 \times 5$ & 1000 \\
\hline 65 & $\mathrm{~N}(1,1)$ & $\mathrm{N}(1,1)$ & $\mathrm{N}(1,1)$ & $\mathrm{N}(0.262,0.04)$ & $3 \times 4$ & 500 \\
\hline 66 & $\mathrm{~N}(1,1)$ & $\mathrm{N}(1,1)$ & $\mathrm{N}(1,1)$ & $\mathrm{N}(0.262,0.4)$ & $3 \times 4$ & 500 \\
\hline 67 & $\mathrm{~N}(1,1)$ & $\mathrm{N}(1,1)$ & $\mathrm{N}(1,1)$ & $\mathrm{N}(0.262,0.04)$ & $3 \times 4$ & 1000 \\
\hline 68 & $\mathrm{~N}(1,1)$ & $\mathrm{N}(1,1)$ & $\mathrm{N}(1,1)$ & $\mathrm{N}(0.262,0.4)$ & $3 \times 4$ & 1000 \\
\hline 69 & $\mathrm{~N}(1,1)$ & $\mathrm{N}(1,1)$ & $\mathrm{N}(1,1)$ & $\mathrm{N}(0.262,0.04)$ & $4 \times 5$ & 500 \\
\hline 70 & $\mathrm{~N}(1,1)$ & $\mathrm{N}(1,1)$ & $\mathrm{N}(1,1)$ & $\mathrm{N}(0.262,0.4)$ & $4 \times 5$ & 500 \\
\hline 71 & $\mathrm{~N}(1,1)$ & $\mathrm{N}(1,1)$ & $\mathrm{N}(1,1)$ & $\mathrm{N}(0.262,0.04)$ & $4 \times 5$ & 1000 \\
\hline 72 & $\mathrm{~N}(1,1)$ & $\mathrm{N}(1,1)$ & $\mathrm{N}(1,1)$ & $\mathrm{N}(0.262,0.4)$ & $4 \times 5$ & 1000 \\
\hline
\end{tabular}

random samples are given through columns $7-9$. The Wald test statistic for the individual parameters should be distributed as chi-square with a mean of $K-2$. As seen in these columns, the mean values of the individual test statistic are approximately $K-2$ for small degrees of freedom (i.e., for a $3 \times 4$ table), whereas they are smaller than $K-2$ in larger tables (i.e., for a $4 \times 5$ table).

Through columns 10-12, the percentage of rejections of proposed individual test statistics under the $\chi_{K-2}^{2}$ distribution are given. As seen in these columns, when the number of degrees of freedom is small (i.e., for a $3 \times 4$ table), type I error rates of the proposed test statistic are around the 0.05 level. However, the type I error rates of the individual test statistic are very conservative in the situations where the number of degrees of freedom is large (i.e., for a $4 \times 5$ table).

The appropriateness of the chi-square approximation of the individual version of the proposed test statistic was investigated using pdf, cdf and Q-Q plots. All of the graphs are not given here to save space; only the graphical results related to scenario number 18 and 20 are given in Figure 3 and Figure 4, respectively.

In Figure 3, the solid lines represent the $\chi_{2}^{2}$ distribution and the dotted lines represent the individual version of the proposed Wald test statistic which are calculated from 1,000 replications. Note that for the $18^{\text {th }}$ scenario, there are two individual tests (i.e., for variables $X_{1}$ and $X_{2}$, respectively) and the degrees of freedom is equal to 2 for the individual Wald tests. As seen from that figure, for both variables, the solid and dotted lines overlap, and in the Q-Q plots, the values of the individual version of the proposed Wald test statistic lie on the $45^{\circ}$ line.

In Figure 4, the solid lines represent the $\chi_{3}^{2}$ distribution and the dotted lines represent the individual version of the proposed Wald test statistic which are calculated from 1,000 replications. Note that for the $20^{t h}$ scenario, there are three individual tests (i.e., for variables $X_{1}, X_{2}$ and $X_{3}$, respectively) and the degrees of freedom is equal to 3 for the individual Wald tests. As seen from that figure, for the individual tests, the solid and dotted lines do not overlap. Also in the Q-Q plots, the values of the individual version of the proposed Wald test statistic do not lie on the $45^{\circ}$ line. Therefore, we can say that the chi-square approximation is labile when the number of independent variables (so the degrees of freedom) increases.

After examining the probability density function, cumulative distribution function and Q-Q plots of the individual version of the proposed test statistic, we concluded that under $H_{0}$, the chi-square approximation is feasible for the individual version of the proposed Wald test statistic, provided that the number of degrees of freedom is not so large for the $R \times C$ table.

The results of the second simulation study are given in Table 5. 
Table 4. Results of the first simulation study

\begin{tabular}{|c|c|c|c|c|c|c|c|c|c|c|c|}
\hline \multirow{3}{*}{$\begin{array}{l}\text { Scenario } \\
\text { Number } \\
1\end{array}$} & \multirow{3}{*}{$\frac{R \times C}{3 \times 4}$} & \multicolumn{2}{|c|}{ Mean Values of Omn. } & \multicolumn{2}{|c|}{ Type I error of Omn. } & \multirow{2}{*}{\multicolumn{3}{|c|}{$\begin{array}{c}\text { Mean Values of } \\
\text { Prop. test (Ind.) }\end{array}$}} & \multirow{2}{*}{\multicolumn{3}{|c|}{$\begin{array}{l}\text { Type I error of } \\
\text { Prop. test (Ind.) }\end{array}$}} \\
\hline & & \multirow{2}{*}{$\begin{array}{c}\text { LR test } \\
3.968\end{array}$} & \multirow{2}{*}{$\begin{array}{c}\text { Prop. test } \\
3.863\end{array}$} & \multirow{2}{*}{$\begin{array}{c}\text { LR test } \\
0.043\end{array}$} & \multirow{2}{*}{$\begin{array}{l}\text { Prop. test } \\
0.035\end{array}$} & & & & & & \\
\hline & & & & & & 1.904 & 1.954 & & 0.039 & 0.051 & \\
\hline 2 & $3 \times 4$ & 3.986 & 3.929 & 0.056 & 0.049 & 2.005 & 1.909 & & 0.064 & 0.037 & \\
\hline 3 & $4 \times 5$ & 9.270 & 8.725 & 0.059 & 0.031 & 1.944 & 1.961 & 1.942 & 0.017 & 0.014 & 0.007 \\
\hline 4 & $4 \times 5$ & 9.101 & 8.796 & 0.047 & 0.037 & 1.959 & 1.975 & 1.993 & 0.013 & 0.020 & 0.017 \\
\hline 5 & $3 \times 4$ & 4.083 & 4.004 & 0.054 & 0.042 & 1.948 & 1.965 & & 0.044 & 0.041 & \\
\hline 6 & $3 \times 4$ & 4.068 & 4.032 & 0.057 & 0.055 & 1.999 & 2.035 & & 0.045 & 0.059 & \\
\hline 7 & $4 \times 5$ & 9.195 & 8.634 & 0.052 & 0.031 & 1.920 & 1.902 & 1.886 & 0.014 & 0.009 & 0.009 \\
\hline 8 & $4 \times 5$ & 9.291 & 9.044 & 0.073 & 0.057 & 2.044 & 2.057 & 1.947 & 0.020 & 0.022 & 0.016 \\
\hline 9 & $3 \times 4$ & 4.045 & 3.935 & 0.063 & 0.050 & 1.943 & 1.966 & & 0.046 & 0.041 & \\
\hline 10 & $3 \times 4$ & 4.170 & 4.116 & 0.058 & 0.055 & 2.043 & 2.054 & & 0.052 & 0.054 & \\
\hline 11 & $4 \times 5$ & 8.568 & 7.820 & 0.032 & 0.012 & 1.651 & 1.656 & 1.658 & 0.007 & 0.003 & 0.010 \\
\hline 12 & $4 \times 5$ & 9.226 & 8.636 & 0.059 & 0.036 & 1.943 & 1.887 & 1.907 & 0.012 & 0.005 & 0.014 \\
\hline 13 & $3 \times 4$ & 3.921 & 3.730 & 0.042 & 0.031 & 1.975 & 1.788 & & 0.048 & 0.027 & \\
\hline 14 & $3 \times 4$ & 4.113 & 3.964 & 0.052 & 0.045 & 2.049 & 1.932 & & 0.053 & 0.042 & \\
\hline 15 & $4 \times 5$ & 9.044 & 8.424 & 0.053 & 0.032 & 1.973 & 1.940 & 2.026 & 0.017 & 0.007 & 0.018 \\
\hline 16 & $4 \times 5$ & 8.958 & 8.568 & 0.044 & 0.030 & 1.959 & 1.876 & 1.946 & 0.017 & 0.015 & 0.018 \\
\hline 17 & $3 \times 4$ & 3.953 & 3.891 & 0.042 & 0.037 & 1.879 & 1.995 & & 0.035 & 0.047 & \\
\hline 18 & $3 \times 4$ & 4.066 & 4.028 & 0.051 & 0.044 & 2.074 & 2.044 & & 0.060 & 0.056 & \\
\hline 19 & $4 \times 5$ & 8.875 & 8.543 & 0.041 & 0.026 & 1.974 & 1.891 & 1.815 & 0.015 & 0.012 & 0.010 \\
\hline 20 & $4 \times 5$ & 8.999 & 8.837 & 0.050 & 0.041 & 1.991 & 2.000 & 1.985 & 0.019 & 0.020 & 0.015 \\
\hline 21 & $3 \times 4$ & 4.051 & 3.965 & 0.060 & 0.049 & 1.936 & 2.018 & & 0.039 & 0.052 & \\
\hline 22 & $3 \times 4$ & 3.944 & 3.906 & 0.045 & 0.038 & 1.957 & 1.924 & & 0.049 & 0.042 & \\
\hline 23 & $4 \times 5$ & 8.980 & 8.403 & 0.051 & 0.032 & 1.801 & 1.840 & 1.828 & 0.006 & 0.005 & 0.010 \\
\hline 24 & $4 \times 5$ & 9.094 & 8.790 & 0.046 & 0.032 & 2.017 & 1.911 & 1.962 & 0.016 & 0.017 & 0.014 \\
\hline 25 & $3 \times 4$ & 3.894 & 3.677 & 0.048 & 0.036 & 1.890 & 1.782 & & 0.032 & 0.035 & \\
\hline 26 & $3 \times 4$ & 4.106 & 3.940 & 0.049 & 0.037 & 2.083 & 1.929 & & 0.069 & 0.037 & \\
\hline 27 & $4 \times 5$ & 8.828 & 8.080 & 0.036 & 0.015 & 1.908 & 1.753 & 1.896 & 0.013 & 0.005 & 0.014 \\
\hline 28 & $4 \times 5$ & 9.065 & 8.562 & 0.054 & 0.032 & 1.932 & 1.905 & 1.924 & 0.018 & 0.011 & 0.017 \\
\hline 29 & $3 \times 4$ & 4.156 & 4.059 & 0.061 & 0.055 & 2.095 & 2.010 & & 0.058 & 0.052 & \\
\hline 30 & $3 \times 4$ & 4.026 & 3.983 & 0.051 & 0.049 & 2.003 & 1.976 & & 0.057 & 0.047 & \\
\hline 31 & $4 \times 5$ & 9.447 & 9.052 & 0.059 & 0.042 & 2.020 & 2.007 & 1.989 & 0.012 & 0.012 & 0.019 \\
\hline 32 & $4 \times 5$ & 9.046 & 8.850 & 0.049 & 0.040 & 1.966 & 1.938 & 2.056 & 0.014 & 0.019 & 0.024 \\
\hline 33 & $3 \times 4$ & 4.215 & 4.113 & 0.063 & 0.058 & 2.110 & 2.090 & & 0.055 & 0.057 & \\
\hline 34 & $3 \times 4$ & 4.039 & 3.994 & 0.053 & 0.049 & 2.067 & 1.938 & & 0.053 & 0.049 & \\
\hline 35 & $4 \times 5$ & 9.264 & 8.680 & 0.058 & 0.033 & 2.015 & 1.898 & 1.857 & 0.010 & 0.007 & 0.012 \\
\hline 36 & $4 \times 5$ & 8.993 & 8.701 & 0.046 & 0.031 & 2.012 & 2.010 & 1.932 & 0.020 & 0.017 & 0.009 \\
\hline
\end{tabular}

LR test: The Likelihood Ratio Test Statistic

Prop. test: The Proposed Wald Test Statistic

Omn.: Omnibus

Ind.: Individual

In Table 5, the mean values of the LR and the proposed test statistics that were computed from 1,000 random samples under the alternative hypotheses are given in columns 5 and 6 . As seen in these columns, the mean values of the proposed Wald test statistic are always smaller than the likelihood ratio test statistics. The seventh and eighth columns of Table 5 give the percentage of times the tests reject the null hypothesis when the null hypothesis is false. An increase in sample size has a positive effect on the power of both tests. Moreover, when the degree of non-proportionality (i.e., the standard deviation of $\beta_{i j}$ ) is increased, the powers of the proposed test and LR test increase. As expected, power of the proposed test is always smaller than or equal to the like- lihood ratio test and tests become equivalent as the sample size increases (e.g., $\mathrm{n}=1,000$ ).

\section{AN EXAMPLE}

The "Attitudes Toward Working Mothers General Survey" (from the Long's book [21, p. 125]) data are used to illustrate the methods used for this study. This data contains information from the 2,293 respondents on the attitudes toward working mothers. The survey is conducted in 1977 and 1989, and the respondents are asked to evaluate the following statement: "A working mother can establish just as warm and secure relationship with her children as 

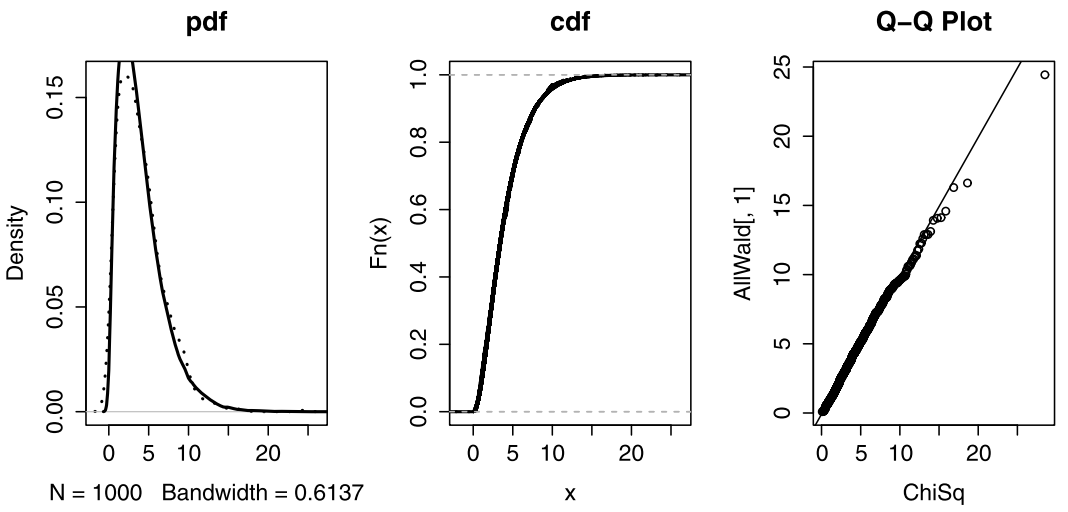

(a)

pdf

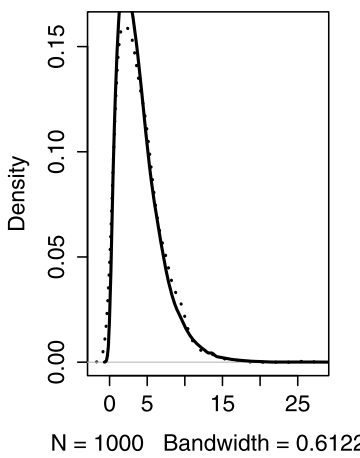

cdf

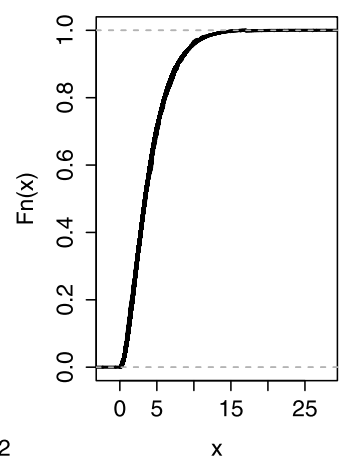

Q-Q Plot

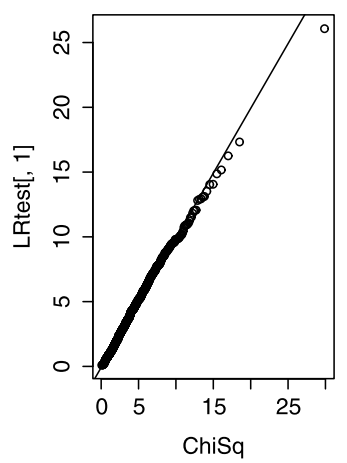

Figure 1. The $p d f$, $c d f$, and $Q-Q$ plots of the (a) omnibus version of the proposed test statistic vs. $\chi_{J(K-2)}^{2}$ distribution and (b) LR test vs. $\chi_{J(K-2)}^{2}$ distribution for the scenario number 18.

a mother who does not work". Responses are coded in the variable WARM as: $1=$ Strongly Disagree $(\mathrm{SD}) ; 2=$ Disagree (D); $3=$ Agree (A); and $4=$ Strongly Agree (SA). The goal of this study was to determine whether the survey year (YR89), sex of the respondent (MALE), race of the respondent (WHITE), age of the respondent (AGE) and education year of the respondent (EDU) are associated with the attitudes toward working mothers. The data code sheet is given in Table 6.

Suppose that we want to determine the effects of the survey year (YR89), sex of the respondent (MALE), race of the respondent (WHITE), age of the respondent (AGE) and education year of the respondent (EDU) on the attitudes toward working mothers (WARM) using the adjacent category model. Before proceeding with any modelling, we must check the proportionality assumption using the proposed method given in Section 5. Table 7 gives the results of the LR test and the proposed test of proportionality for this data.

According to both LR and the omnibus version of the proposed test results, at least one factor in the ACM does not satisfy the proportionality assumption $(p<0.001$ and $p<0.001$, respectively). When we examine the individual Wald test statistics of these factors, we observe that the survey year (YR89), sex of the respondent (MALE), and age of the respondent (AGE) violate the proportionality assumption. Therefore, we must construct a partial proportional adjacent category model for this data.

In order to determine the parameter estimations in the PPACM, we utilize the relationships between the adjacent category model, the non-proportional adjacent category model, and the multinomial logistic regression model, which are given in Sections 3.7 and 3.8. Because the adjacent category model and the non-proportional adjacent category model is a constrained version of the multinomial logistic regression model (See Equations (16) and (23)), we start by fitting a multinomial logistic regression in order to get the NPACM, ACM and PPACM results.

Table 8 gives the results of the multinomial logistic regression model for the attitudes toward working mothers data. The non-proportional adjacent category model is the constrained version of the MLRM.

Table 9 gives the parameter and standard error estimates of the non-proportional adjacent category model, which is directly obtained from the MLRM using the constraints in Equation (24). As seen in Tables 8 and 9, these models have equal log-likelihoods.

In the non-proportional adjacent category model, the first logit estimation results of the coefficients should be the same as the multinomial logistic regression results. From Equation 

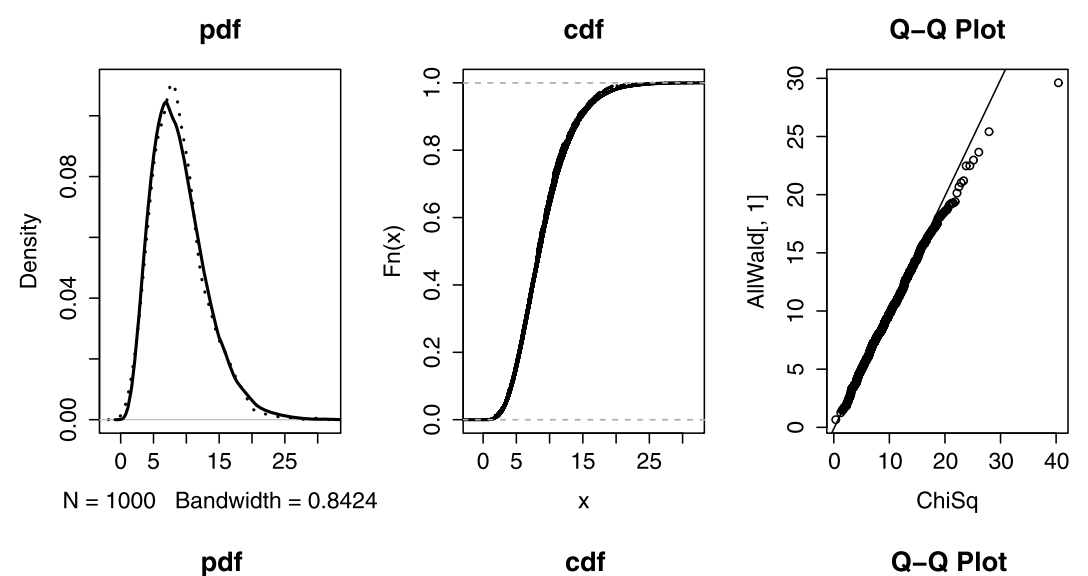

(a)

\section{pdf}

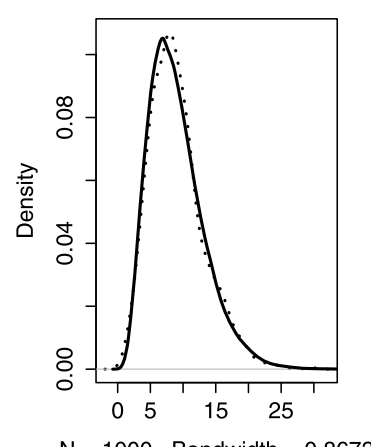

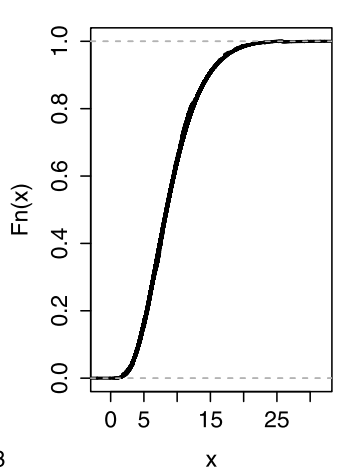

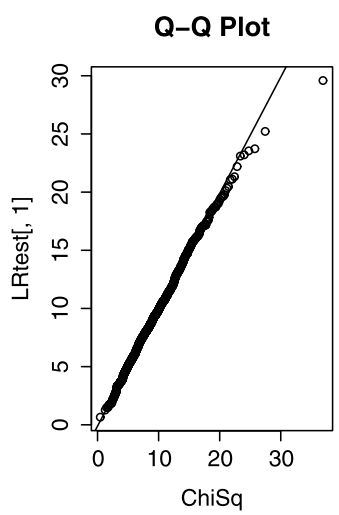

Figure 2. The $p d f$, cdf, and $Q-Q$ plots of the (a) omnibus version of the proposed test statistic vs. $\chi_{J(K-2)}^{2}$ distribution and (b) LR test vs. $\chi_{J(K-2)}^{2}$ distribution for the scenario number 20.

(24), the second logit estimates of the NPACM results are found by taking the difference of the logit 2 of the MLRM results from the logit 1 , and the third logit results are found by taking the difference of logit 3 of the MLRM results from the logit 2. For example in Table 9, the coefficient estimate of the WHITE in the third logit can be found as $-0.8283-$ $(-0.5321)=-0.2962$.

The adjacent category model is also a constrained version of the multinomial logistic regression model. In order to get the parameter estimates in the adjacent category model, first we fit a constrained multinomial logistic regression model (using MLE methods) with constraint $\beta_{k}=k \beta$, which is given in Equation (17). Table 10 gives the maximum likelihood estimation results for the constrained multinomial logistic regression model.

Note that in Table 10, since we applied the constraint $\beta_{k}=k \beta$, the coefficient estimates in the second logit are twice the coefficient estimates in the first logit, and the coefficient estimates in the third logit are treble of the coefficient estimates in the third logit. For example, the coefficient estimate for the race of the respondent (WHITE) in the third logit will be equal to $3 \times-0.2307=-0.6922$. Because the adjacent category model possesses the property that the independent variables have equal effects on the outcome across the adjacent categories, the effects of the independent vari- ables do not vary across logits and they are equal to the coefficients which are given in the first logit of Table 10. Such as the coefficient estimate of survey year (YR89) and the coefficient estimate of education year of the respondent (EDU) for the adjacent category model will be equal to 0.3463 and 0.0508 , respectively.

After fitting the multinomial logistic regression with constraint $\beta_{k}=k \beta$, and getting the regression coefficient estimates, now we can get the intercept terms for the adjacent category model using the property $\beta_{0 k}=\sum_{i=1}^{k} \alpha_{i}$. For example, the intercept term for the first logit of adjacent category model (which is 1.2817) will be equal to the intercept of the first logit of the constrained multinomial logistic regression model in Table 11. The second intercept term of the adjacent category model is found by taking the difference of intercept of the second logit and the first logit, which is $1.6779-1.2817=0.3962$. Similarly, the intercept term for the third logit will be $1.0325-1.6779=-0.6454$. Therefore, we get the parameter and standard error estimates of the adjacent category model as given in Table 11.

We have found that the survey year (YR89), sex of the respondent (MALE), and age of the respondent (AGE) violate the proportionality assumption. Thus, when we apply the constraints in Equation (17) for the race (WHITE) and education year of the respondent while not apply- 

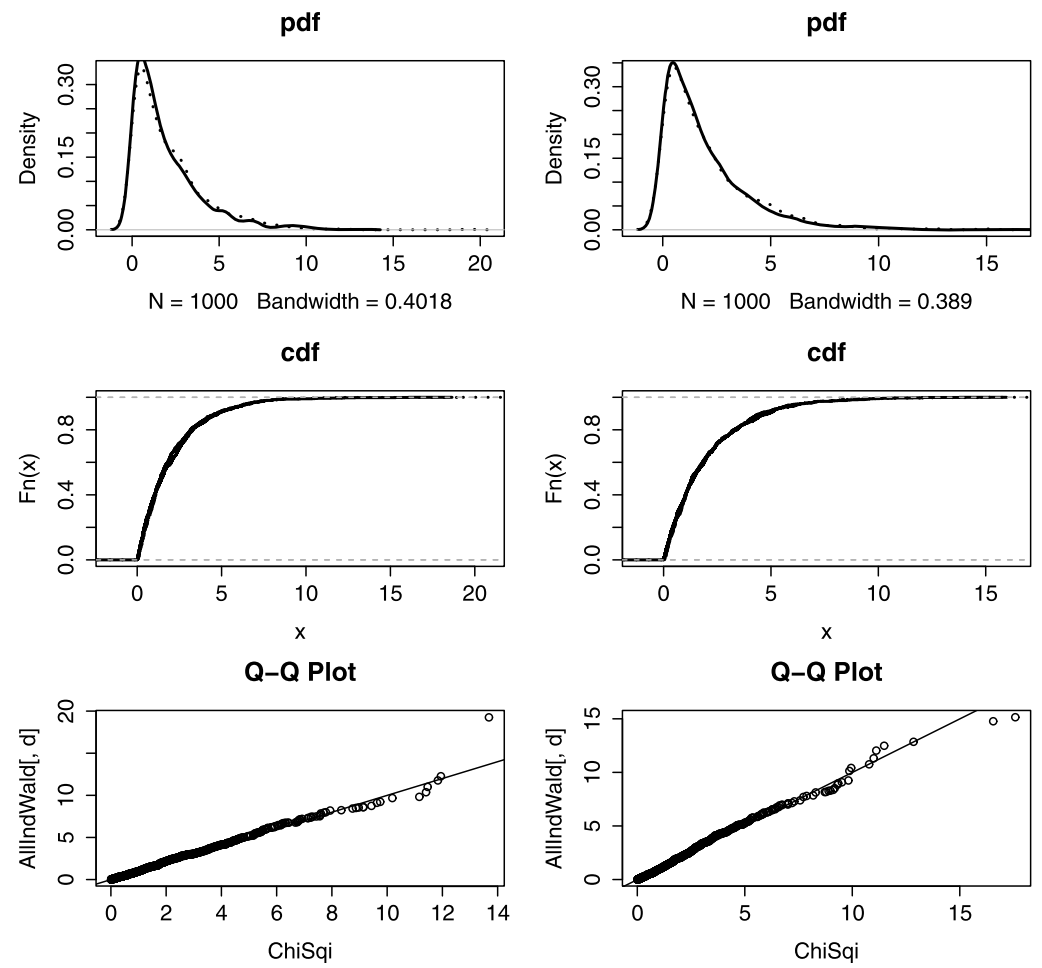

Figure 3. The $p d f$, $c d f$, and $Q-Q$ plots of the individual version of the proposed test statistic vs. $\chi_{K-2}^{2}$ distribution for the scenario number 18.
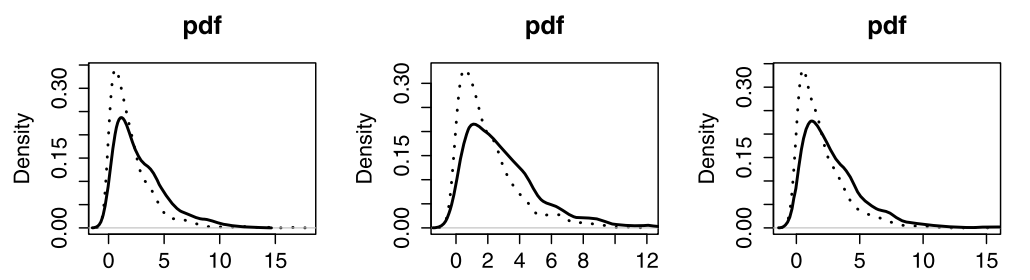

$\mathrm{N}=1000$ Bandwidth $=0.3445$

$\mathrm{N}=1000$ Bandwidth $=0.3471$

$\mathrm{N}=1000$ Bandwidth $=0.3823$
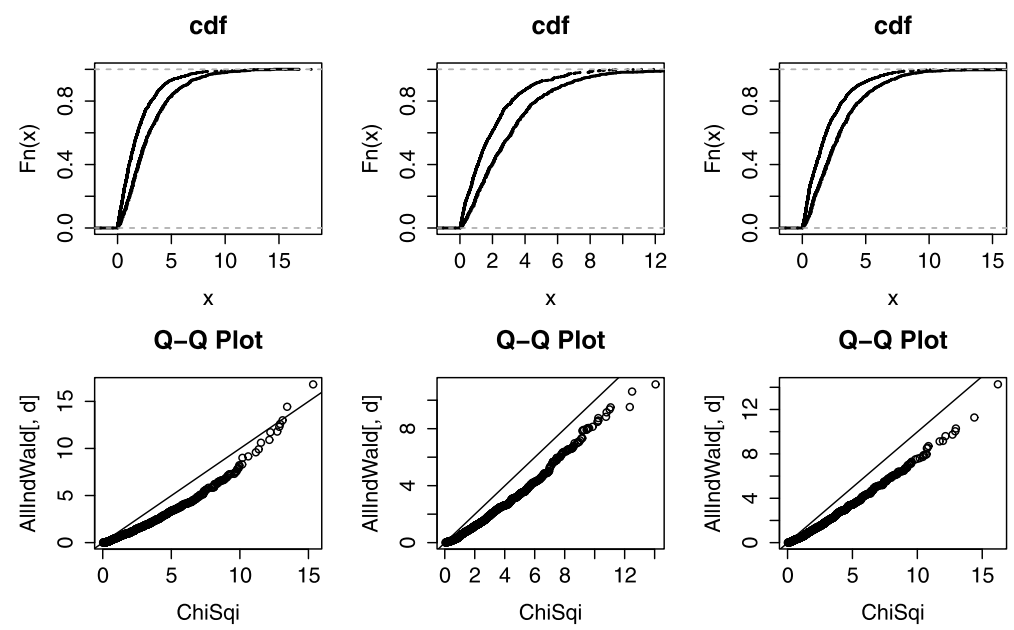

Figure 4. The $p d f$, $c d f$, and $Q-Q$ plots of the individual version of the proposed test statistic vs. $\chi_{K-2}^{2}$ distribution for the scenario number 20. 
Table 5. Results of the second simulation study

\begin{tabular}{|c|c|c|c|c|c|c|c|}
\hline \multirow{2}{*}{$\begin{array}{l}\text { Scenario } \\
\text { Number }\end{array}$} & \multirow{2}{*}{$\begin{array}{c}\text { Distribution of } \\
\beta_{i j}\end{array}$} & \multirow[b]{2}{*}{$R \times C$} & \multirow[b]{2}{*}{ Sample Size } & \multicolumn{2}{|c|}{ Mean values of } & \multicolumn{2}{|c|}{ Power of } \\
\hline & & & & LR test & Prop. test & LR test & Prop. test \\
\hline 1 & $\mathrm{~N}(0,0.02)$ & $3 \times 4$ & 300 & 4.097 & 3.864 & 0.048 & 0.027 \\
\hline 2 & $\mathrm{~N}(0,0.2)$ & $3 \times 4$ & 300 & 7.035 & 6.131 & 0.244 & 0.195 \\
\hline 3 & $\mathrm{~N}(0,0.02)$ & $3 \times 4$ & 500 & 4.194 & 4.059 & 0.058 & 0.043 \\
\hline 4 & $\mathrm{~N}(0,0.2)$ & $3 \times 4$ & 500 & 11.465 & 10.161 & 0.596 & 0.509 \\
\hline 5 & $\mathrm{~N}(0,0.02)$ & $4 \times 5$ & 300 & 9.818 & 9.045 & 0.082 & 0.045 \\
\hline 6 & $\mathrm{~N}(0,0.2)$ & $4 \times 5$ & 300 & 112.220 & 69.883 & 1.000 & 0.997 \\
\hline 7 & $\mathrm{~N}(0,0.02)$ & $4 \times 5$ & 500 & 11.754 & 10.977 & 0.177 & 0.126 \\
\hline 8 & $\mathrm{~N}(0,0.2)$ & $4 \times 5$ & 500 & 190.540 & 110.612 & 1.000 & 0.993 \\
\hline 9 & $\mathrm{~N}(0.182,0.03)$ & $3 \times 4$ & 500 & 4.826 & 4.420 & 0.102 & 0.068 \\
\hline 10 & $\mathrm{~N}(0.182,0.3)$ & $3 \times 4$ & 500 & 17.483 & 15.510 & 0.864 & 0.815 \\
\hline 11 & $\mathrm{~N}(0.182,0.03)$ & $3 \times 4$ & 1000 & 5.767 & 5.500 & 0.155 & 0.139 \\
\hline 12 & $\mathrm{~N}(0.182,0.3)$ & $3 \times 4$ & 1000 & 37.347 & 32.206 & 1.000 & 1.000 \\
\hline 13 & $\mathrm{~N}(0.182,0.03)$ & $4 \times 5$ & 500 & 24.985 & 22.930 & 0.813 & 0.770 \\
\hline 14 & $\mathrm{~N}(0.182,0.3)$ & $4 \times 5$ & 500 & 379.030 & 135.420 & 1.000 & 0.999 \\
\hline 15 & $\mathrm{~N}(0.182,0.03)$ & $4 \times 5$ & 1000 & 42.883 & 40.203 & 0.992 & 0.990 \\
\hline 16 & $\mathrm{~N}(0.182,0.3)$ & $4 \times 5$ & 1000 & 765.160 & 254.500 & 1.000 & 1.000 \\
\hline 17 & $\mathrm{~N}(0.262,0.04)$ & $3 \times 4$ & 500 & 4.774 & 4.455 & 0.081 & 0.061 \\
\hline 18 & $\mathrm{~N}(0.262,0.4)$ & $3 \times 4$ & 500 & 22.905 & 20.194 & 0.958 & 0.946 \\
\hline 19 & $\mathrm{~N}(0.262,0.04)$ & $3 \times 4$ & 1000 & 5.136 & 4.974 & 0.109 & 0.095 \\
\hline 20 & $\mathrm{~N}(0.262,0.4)$ & $3 \times 4$ & 1000 & 46.093 & 40.133 & 1.000 & 1.000 \\
\hline 21 & $\mathrm{~N}(0.262,0.04)$ & $4 \times 5$ & 500 & 37.893 & 33.171 & 0.984 & 0.979 \\
\hline 22 & $\mathrm{~N}(0.262,0.4)$ & $4 \times 5$ & 500 & 271.280 & 89.328 & 1.000 & 1.000 \\
\hline 23 & $\mathrm{~N}(0.262,0.04)$ & $4 \times 5$ & 1000 & 69.052 & 61.049 & 1.000 & 1.000 \\
\hline 24 & $\mathrm{~N}(0.262,0.4)$ & $4 \times 5$ & 1000 & 517.440 & 148.932 & 1.000 & 1.000 \\
\hline 25 & $\mathrm{~N}(0,0.02)$ & $3 \times 4$ & 300 & 4.242 & 3.960 & 0.058 & 0.038 \\
\hline 26 & $\mathrm{~N}(0,0.2)$ & $3 \times 4$ & 300 & 6.636 & 6.031 & 0.219 & 0.147 \\
\hline 27 & $\mathrm{~N}(0,0.02)$ & $3 \times 4$ & 500 & 4.219 & 3.997 & 0.057 & 0.045 \\
\hline 28 & $\mathrm{~N}(0,0.2)$ & $3 \times 4$ & 500 & 9.858 & 8.575 & 0.472 & 0.383 \\
\hline 29 & $\mathrm{~N}(0,0.02)$ & $4 \times 5$ & 300 & 9.777 & 8.934 & 0.078 & 0.034 \\
\hline 30 & $\mathrm{~N}(0,0.2)$ & $4 \times 5$ & 300 & 134.180 & 58.572 & 1.000 & 0.877 \\
\hline 31 & $\mathrm{~N}(0,0.02)$ & $4 \times 5$ & 500 & 11.155 & 10.265 & 0.115 & 0.073 \\
\hline 32 & $\mathrm{~N}(0,0.2)$ & $4 \times 5$ & 500 & 222.290 & 104.758 & 1.000 & 0.983 \\
\hline 33 & $\mathrm{~N}(0.182,0.03)$ & $3 \times 4$ & 500 & 4.4279 & 4.2523 & 0.071 & 0.059 \\
\hline 34 & $\mathrm{~N}(0.182,0.3)$ & $3 \times 4$ & 500 & 16.402 & 14.283 & 0.831 & 0.778 \\
\hline 35 & $\mathrm{~N}(0.182,0.03)$ & $3 \times 4$ & 1000 & 4.6166 & 4.5136 & 0.078 & 0.067 \\
\hline 36 & $\mathrm{~N}(0.182,0.3)$ & $3 \times 4$ & 1000 & 32.541 & 28.518 & 0.998 & 0.995 \\
\hline 37 & $\mathrm{~N}(0.182,0.03)$ & $4 \times 5$ & 500 & 23.464 & 22.091 & 0.767 & 0.724 \\
\hline 38 & $\mathrm{~N}(0.182,0.3)$ & $4 \times 5$ & 500 & 237.310 & 109.440 & 1.000 & 1.000 \\
\hline 39 & $\mathrm{~N}(0.182,0.03)$ & $4 \times 5$ & 1000 & 38.271 & 36.821 & 0.986 & 0.985 \\
\hline 40 & $\mathrm{~N}(0.182,0.3)$ & $4 \times 5$ & 1000 & 479.750 & 210.370 & 1.000 & 1.000 \\
\hline 41 & $\mathrm{~N}(0.262,0.04)$ & $3 \times 4$ & 500 & 4.751 & 4.517 & 0.083 & 0.066 \\
\hline 42 & $\mathrm{~N}(0.262,0.4)$ & $3 \times 4$ & 500 & 21.174 & 17.801 & 0.945 & 0.922 \\
\hline 43 & $\mathrm{~N}(0.262,0.04)$ & $3 \times 4$ & 1000 & 5.163 & 5.020 & 0.123 & 0.102 \\
\hline 44 & $\mathrm{~N}(0.262,0.4)$ & $3 \times 4$ & 1000 & 43.437 & 36.722 & 1.000 & 0.999 \\
\hline 45 & $\mathrm{~N}(0.262,0.04)$ & $4 \times 5$ & 500 & 38.547 & 35.969 & 0.988 & 0.987 \\
\hline 46 & $\mathrm{~N}(0.262,0.4)$ & $4 \times 5$ & 500 & 145.360 & 64.942 & 1.000 & 0.999 \\
\hline 47 & $\mathrm{~N}(0.262,0.04)$ & $4 \times 5$ & 1000 & 69.353 & 65.660 & 1.000 & 1.000 \\
\hline 48 & $\mathrm{~N}(0.262,0.4)$ & $4 \times 5$ & 1000 & 261.680 & 101.837 & 1.000 & 1.000 \\
\hline
\end{tabular}

ing any constraints to the rest of the variables (YR89, MALE, AGE), we get the parameter estimates of partial version of the adjacent category model. The partial proportional adjacent category model results are given in Table 12 .
From Table 12 we can see that the race and education year of the respondents have the same effect across the adjacent logits. White respondents tend to agree more by $\exp (-0.2304)=0.7942$ times (which means they do not agree) when compared to the non-white respondents, while 
Table 5. (Continued)

\begin{tabular}{|c|c|c|c|c|c|c|c|}
\hline \multirow{2}{*}{$\begin{array}{l}\text { Scenario } \\
\text { Number }\end{array}$} & \multirow{2}{*}{$\begin{array}{c}\text { Distribution of } \\
\beta_{i j}\end{array}$} & \multirow[b]{2}{*}{$R \times C$} & \multirow[b]{2}{*}{ Sample Size } & \multicolumn{2}{|c|}{ Mean values of } & \multicolumn{2}{|c|}{ Power of } \\
\hline & & & & LR test & Prop. test & LR test & Prop. test \\
\hline 49 & $\mathrm{~N}(0,0.02)$ & $3 \times 4$ & 300 & 3.694 & 3.378 & 0.033 & 0.019 \\
\hline 50 & $\mathrm{~N}(0,0.2)$ & $3 \times 4$ & 300 & 4.567 & 4.070 & 0.078 & 0.039 \\
\hline 51 & $\mathrm{~N}(0,0.02)$ & $3 \times 4$ & 500 & 3.970 & 3.665 & 0.040 & 0.027 \\
\hline 52 & $\mathrm{~N}(0,0.2)$ & $3 \times 4$ & 500 & 6.112 & 5.380 & 0.172 & 0.103 \\
\hline 53 & $\mathrm{~N}(0,0.02)$ & $4 \times 5$ & 300 & 8.227 & 7.676 & 0.031 & 0.021 \\
\hline 54 & $\mathrm{~N}(0,0.2)$ & $4 \times 5$ & 300 & 82.892 & 17.738 & 1.000 & 0.574 \\
\hline 55 & $\mathrm{~N}(0,0.02)$ & $4 \times 5$ & 500 & 9.498 & 8.888 & 0.072 & 0.047 \\
\hline 56 & $\mathrm{~N}(0,0.2)$ & $4 \times 5$ & 500 & 131.635 & 23.729 & 1.000 & 0.917 \\
\hline 57 & $\mathrm{~N}(0.182,0.03)$ & $3 \times 4$ & 500 & 4.291 & 3.990 & 0.058 & 0.041 \\
\hline 58 & $\mathrm{~N}(0.182,0.3)$ & $3 \times 4$ & 500 & 9.696 & 8.687 & 0.444 & 0.371 \\
\hline 59 & $\mathrm{~N}(0.182,0.03)$ & $3 \times 4$ & 1000 & 4.595 & 4.408 & 0.088 & 0.071 \\
\hline 60 & $\mathrm{~N}(0.182,0.3)$ & $3 \times 4$ & 1000 & 18.213 & 16.163 & 0.886 & 0.841 \\
\hline 61 & $\mathrm{~N}(0.182,0.03)$ & $4 \times 5$ & 500 & 19.711 & 18.394 & 0.584 & 0.538 \\
\hline 62 & $\mathrm{~N}(0.182,0.3)$ & $4 \times 5$ & 500 & 365.570 & 158.990 & 1.000 & 0.997 \\
\hline 63 & $\mathrm{~N}(0.182,0.03)$ & $4 \times 5$ & 1000 & 33.708 & 31.942 & 0.962 & 0.955 \\
\hline 64 & $\mathrm{~N}(0.182,0.3)$ & $4 \times 5$ & 1000 & 741.760 & 315.250 & 1.000 & 1.000 \\
\hline 65 & $\mathrm{~N}(0.262,0.04)$ & $3 \times 4$ & 500 & 4.676 & 4.306 & 0.098 & 0.061 \\
\hline 66 & $\mathrm{~N}(0.262,0.4)$ & $3 \times 4$ & 500 & 13.387 & 11.371 & 0.705 & 0.603 \\
\hline 67 & $\mathrm{~N}(0.262,0.04)$ & $3 \times 4$ & 1000 & 4.580 & 4.352 & 0.083 & 0.062 \\
\hline 68 & $\mathrm{~N}(0.262,0.4)$ & $3 \times 4$ & 1000 & 25.454 & 21.705 & 0.979 & 0.969 \\
\hline 69 & $\mathrm{~N}(0.262,0.04)$ & $4 \times 5$ & 500 & 36.565 & 34.045 & 0.975 & 0.971 \\
\hline 70 & $\mathrm{~N}(0.262,0.4)$ & $4 \times 5$ & 500 & 294.180 & 97.420 & 1.000 & 1.000 \\
\hline 71 & $\mathrm{~N}(0.262,0.04)$ & $4 \times 5$ & 1000 & 67.694 & 63.719 & 1.000 & 1.000 \\
\hline 72 & $\mathrm{~N}(0.262,0.4)$ & $4 \times 5$ & 1000 & 586.850 & 175.330 & 1.000 & 1.000 \\
\hline
\end{tabular}

LR test: The Likelihood Ratio Test Statistic

Prop. test: The Proposed Wald Test Statistic

Table 6. Data code sheet for the variables in the attitudes toward working mothers general survey data

\begin{tabular}{|c|c|c|c|}
\hline Variable & Description & Codes/Values & Name \\
\hline 1 & $\begin{array}{l}\text { Response to: "A working mother } \\
\text { can establish just as warm } \\
\text { and secure relationship with } \\
\text { her children as a mother } \\
\text { who does not work" }\end{array}$ & $\begin{array}{l}\text { 1: Strongly Disagree (SD) } \\
\text { 2: Disagree (D) } \\
\text { 3: Agree (A) } \\
\text { 4: Strongly Agree (SA) }\end{array}$ & WARM \\
\hline 2 & Survey Year & $\begin{array}{l}\text { 1: } 1989 \\
0: 1977^{\dagger}\end{array}$ & YR89 \\
\hline 3 & Sex of the respondent & $\begin{array}{l}\text { 1: Male } \\
\text { 0: Female }{ }^{\dagger}\end{array}$ & MALE \\
\hline 4 & Race of the respondent & $\begin{array}{l}\text { 1: White } \\
\text { 0: Non-white }\end{array}$ & WHITE \\
\hline 5 & Age of the respondent & Age in years & AGE \\
\hline 6 & Education year of the respondent & Education in years & EDU \\
\hline
\end{tabular}

with an increase of 1 year in education, respondents tend to agree more with odds of $\exp (0.0518)=1.05$. The survey year, sex of the respondents and age of respondents have different effects across the logits. The coefficients of the variables can be interpreted individually; for example, when compared to females, males tend to agree $\exp (0.0786)=$ 1.08 times for the first logit, $\exp (-0.4424)=0.64$ times in the second logit, and $\exp (-0.8776)=0.41$ times for the third logit. In other words, the degree of agreement with the statement decreases for males.

\section{DISCUSSION AND FUTURE WORK}

Prior to fitting any of ordinal logistic regression models, it is important to check whether the assumption of proportionality is satisfied by each independent variable. In the proportional odds model, the proportional odds assumption 
Table 7. Results of the $L R$ test and the proposed test of proportionality for the attitudes toward working mothers data

\begin{tabular}{lccc}
\hline \hline & Test Statistic & df & p value \\
\hline Likelihood ratio test & 47.969 & 10 & $p<0.001$ \\
Proposed Wald test (Omnibus) & 46.930 & 10 & $p<0.001$ \\
Proposed Wald test for YR89 & 10.811 & 2 & 0.0045 \\
Proposed Wald test for MALE & 24.689 & 2 & $p<0.001$ \\
Proposed Wald test for WHITE & 1.188 & 2 & 0.5522 \\
Proposed Wald test for AGE & 8.708 & 2 & 0.0129 \\
Proposed Wald test for EDU & 0.953 & 2 & 0.6211 \\
\hline
\end{tabular}

Table 8. Estimation results of the multinomial logistic regression model

\begin{tabular}{|c|c|c|c|c|}
\hline Variable & Coefficient & Std. Error & $\mathrm{z}$ value & p value \\
\hline \multicolumn{5}{|c|}{ Logit 1: Disagree } \\
\hline Intercept & 0.4230 & 0.4287 & 0.99 & 0.324 \\
\hline YR89 & 0.7348 & 0.1656 & 4.44 & $p<0.001$ \\
\hline MALE & 0.0829 & 0.1403 & 0.59 & 0.554 \\
\hline WHITE & -0.4456 & 0.2464 & -1.81 & 0.071 \\
\hline $\mathrm{AGE}$ & -0.0037 & 0.0043 & -0.86 & 0.393 \\
\hline EDU & 0.0703 & 0.0230 & 3.05 & 0.002 \\
\hline \multicolumn{5}{|c|}{ Logit 2: Agree } \\
\hline Intercept & 1.0891 & 0.4311 & 2.53 & 0.012 \\
\hline YR89 & 1.0980 & 0.1637 & 6.71 & $p<0.001$ \\
\hline MALE & -0.3623 & 0.1406 & -2.58 & 0.010 \\
\hline WHITE & -0.5321 & 0.2456 & -2.17 & 0.030 \\
\hline $\mathrm{AGE}$ & -0.0246 & 0.0044 & -5.61 & $p<0.001$ \\
\hline EDU & 0.1192 & 0.0236 & 5.06 & $p<0.001$ \\
\hline \multicolumn{5}{|c|}{ Logit 3: Strongly Agree } \\
\hline Intercept & 0.6679 & 0.4929 & 1.36 & 0.175 \\
\hline YR89 & 1.1622 & 0.1811 & 6.42 & $p<0.001$ \\
\hline MALE & -1.2308 & 0.1673 & -7.36 & $p<0.001$ \\
\hline WHITE & -0.8283 & 0.2633 & -3.15 & 0.002 \\
\hline $\mathrm{AGE}$ & -0.0311 & 0.0051 & -6.08 & $p<0.001$ \\
\hline $\mathrm{EDU}$ & 0.1590 & 0.0279 & 5.71 & $p<0.001$ \\
\hline
\end{tabular}

WARM $=$ Strongly Disagree is the baseline outcome

Log-likelihood $=-2825.204$

is checked by the Brant's Wald test statistic [26], and it is performed by the standard Wald test statistic [1] for the continuation ratio model. In the adjacent category logistic regression model, the test of proportionality assumption can be performed with the LR test. However, the LR test is an omnibus test that does not show whether the proportionality assumption is violated for all independent variables or only for some. Therefore, before using the ACM, one needs a valid method to test the proportionality assumption both in an omnibus and individual manner.

Following the idea of Brant [26], we adapted a Wald test that can test the proportionality assumption both in an omnibus and individual manner for checking the proportionality assumption in the ACM. The distribution of the proposed test statistic is examined under the null and alternative hypotheses with a Monte Carlo simulation study. We
Table 9. Estimation results of the non-proportional adjacent category model

\begin{tabular}{lcccc}
\hline \hline Variable & Coefficient & Std. Error & z value & p value \\
\hline Logit 1: Disagree & & & \\
\hline Intercept & 0.4230 & 0.4287 & 0.99 & 0.324 \\
YR89 & 0.7348 & 0.1656 & 4.44 & $p<0.001$ \\
MALE & 0.0829 & 0.1403 & 0.59 & 0.554 \\
WHITE & -0.4456 & 0.2464 & -1.81 & 0.071 \\
AGE & -0.0037 & 0.0043 & -0.85 & 0.393 \\
EDU & 0.0703 & 0.0230 & 3.05 & 0.002 \\
\hline Logit 2: Agree & & & \\
\hline Intercept & 0.6662 & 0.3116 & 2.14 & 0.033 \\
YR89 & 0.3632 & 0.1067 & 3.40 & $p<0.001$ \\
MALE & -0.4453 & 0.1041 & -4.28 & $p<0.001$ \\
WHITE & -0.0865 & 0.1616 & -0.54 & 0.593 \\
AGE & -0.0209 & 0.0033 & -6.43 & $p<0.001$ \\
EDU & 0.0489 & 0.0175 & 2.79 & 0.005 \\
\hline Logit 3: Strongly Agree & & & \\
\hline Intercept & -0.4212 & 0.3732 & -1.13 & 0.259 \\
YR89 & 0.0642 & 0.1229 & 0.52 & 0.601 \\
MALE & -0.8684 & 0.1312 & -6.62 & $p<0.001$ \\
WHITE & -0.2962 & 0.1707 & -1.73 & 0.083 \\
AGE & -0.0065 & 0.0040 & -1.61 & 0.108 \\
EDU & 0.0398 & 0.0220 & 1.81 & 0.070 \\
\hline Log-1ikelitood & --2825.204 & &
\end{tabular}

Log-likelihood $=-2825.204$

Table 10. Estimation results of the constrained multinomial logistic regression model with constraint $\beta_{k}=k \beta$

\begin{tabular}{|c|c|c|c|c|}
\hline Variable & Coefficient & Std. Error & $\mathrm{z}$ value & p value \\
\hline \multicolumn{5}{|c|}{ Logit 1: Disagree } \\
\hline Intercept & 1.2817 & 0.1619 & 7.91 & $p<0.001$ \\
\hline YR89 & 0.3463 & 0.0506 & 6.84 & $p<0.001$ \\
\hline MALE & -0.4354 & 0.0493 & -8.82 & $p<0.001$ \\
\hline WHITE & -0.2307 & 0.0752 & -3.07 & 0.002 \\
\hline $\mathrm{AGE}$ & -0.0127 & 0.0015 & -8.25 & $p<0.001$ \\
\hline EDU & 0.0508 & 0.0083 & 6.15 & $p<0.001$ \\
\hline \multicolumn{5}{|c|}{ Logit 2: Agree } \\
\hline Intercept & 1.6779 & 0.2976 & 5.64 & $p<0.001$ \\
\hline YR89 & 0.6926 & 0.1012 & 6.84 & $p<0.001$ \\
\hline MALE & -0.8708 & 0.0988 & -8.82 & $p<0.001$ \\
\hline WHITE & -0.4615 & 0.1503 & -3.07 & 0.002 \\
\hline AGE & -0.0253 & 0.0031 & -8.25 & $p<0.001$ \\
\hline $\mathrm{EDU}$ & 0.1016 & 0.0165 & 6.15 & $p<0.001$ \\
\hline \multicolumn{5}{|c|}{ Logit 3: Strongly Agree } \\
\hline Intercept & 1.0325 & 0.4374 & 2.36 & 0.018 \\
\hline YR89 & 1.0388 & 0.1519 & 6.84 & $p<0.001$ \\
\hline MALE & -1.3063 & 0.1481 & -8.82 & $p<0.001$ \\
\hline WHITE & -0.6922 & 0.2255 & -3.07 & 0.002 \\
\hline AGE & -0.0379 & 0.0046 & -8.25 & $p<0.001$ \\
\hline EDU & 0.1524 & 0.0248 & 6.15 & $p<0.001$ \\
\hline
\end{tabular}

Log-likelihood $=-2849.189$

examined the appropriateness of chi-square approximation of the proposed test statistic using some graphical methods, and we showed that the proposed test statistic is asymptot- 
Table 11. Estimation results of the adjacent category model

\begin{tabular}{lcccc}
\hline \hline Variable & Coefficient & Std. Error & z value & p value \\
\hline Intercept (Logit 1) & 1.2817 & 0.1619 & 7.9144 & $p<0.001$ \\
Intercept (Logit 2) & 0.3962 & 0.1526 & 2.5965 & 0.009 \\
Intercept (Logit 3) & -0.6454 & 0.1545 & -4.1765 & $p<0.001$ \\
YR89 & 0.3463 & 0.0506 & 6.8420 & $p<0.001$ \\
MALE & -0.4354 & 0.0494 & -8.8203 & $p<0.001$ \\
WHITE & -0.2307 & 0.0752 & -3.0699 & 0.002 \\
AGE & -0.0127 & 0.0015 & -8.2488 & $p<0.001$ \\
EDU & 0.0508 & 0.0083 & 6.1529 & $p<0.001$ \\
\hline
\end{tabular}

Log-likelihood $=-2849.189$

Table 12. Estimation results of the partial-proportional adjacent category model

\begin{tabular}{lcccc}
\hline \hline Variable & Coefficient & Std. Error & $\mathbf{z}$ value & p value \\
\hline Intercept (Logit 1) & 0.4964 & 0.2672 & 1.8580 & 0.063 \\
Intercept (Logit 2) & 0.7454 & 0.2090 & 3.5663 & $p<0.001$ \\
Intercept (Logit 3) & -0.6412 & 0.2242 & -2.8597 & 0.004 \\
WHITE & -0.2304 & 0.0751 & -3.0661 & 0.002 \\
EDU & 0.0518 & 0.0084 & 6.1804 & $p<0.001$ \\
\hline Logit 1: Disagree & & & \\
\hline YR89 & 0.7545 & 0.1639 & 4.6025 & $p<0.001$ \\
MALE & 0.0786 & 0.1400 & 0.5618 & 0.574 \\
AGE & -0.0050 & 0.0041 & -1.2104 & 0.226 \\
\hline Logit 2: Agree & & & \\
\hline YR89 & 0.3585 & 0.1057 & 3.3909 & 0.001 \\
MALE & -0.4424 & 0.1040 & -4.2532 & $p<0.001$ \\
AGE & -0.0206 & 0.0032 & -6.5237 & $p<0.001$ \\
\hline Logit 3: Strongly Agree & & & \\
\hline YR89 & 0.0551 & 0.1216 & 0.4529 & 0.651 \\
MALE & -0.8776 & 0.1308 & -6.7114 & $p<0.001$ \\
AGE & -0.0061 & 0.0039 & -1.5421 & 0.123 \\
\hline Log-likelihod
\end{tabular}

Log-likelihood $=-2826.187$

ically distributed as a chi-square distribution under the null hypothesis.

The likelihood ratio test statistic evaluates the difference in deviances, and it provides an omnibus test of proportionality in the adjacent category model. Therefore, we also compared the results of the omnibus version of the proposed test statistic with the LR test results. According to the simulation results, we observed that type I error rates of the proposed Wald and LR tests are around the desired 0.05 level, while the proposed test statistic has lower type I error rates than the LR test. Type I error rates of these two tests become equivalent as the sample size increases. In terms of power, we observed that the power of the proposed test is always smaller than or equal to the LR test and tests become equivalent as the sample size increases. These findings were consistent with the asymptotic theory, such that under general conditions, the Wald and LR tests are asymptotically equal in distribution, both under the null hypothesis and under the local alternatives [34].

Brant [26] points out that if the degrees of freedom is large, then the Wald test becomes conservative and suffers from the power for the proportional odds model. We also observed that the omnibus and the individual versions of the proposed test statistics tend to be conservative. This conservatism increases especially when the number of parameters tested (i.e., degrees of freedom) increases and when the sample size is small. Consequently, the LR test has higher power than the proposed Wald test, due to the conservatism of the latter method especially in those settings.

The proposed test statistic is an effective way to test the proportionality assumption in the adjacent category model, and it is superior to the likelihood ratio test statistic because it allows both an overall and individual test of proportionality in the ACM. With use of the proposed test statistic, the variables in the ACM that do not obey the proportionality assumption can be determined, and they can be modelled as non-proportional in the partial proportional adjacent category model. Therefore, in this study, we suggest using the proposed test statistic for testing the proportionality assumption in the ACM. The test statistic suggested here has the advantage of requiring only the software for fitting binary logistic regression model plus some basic ma- 
trix manipulations. We also provide the $\mathrm{R}$ routine for the proposed test statistic upon request.

The partial proportional adjacent category model is superior to the adjacent category model for situations where the assumption of proportionality is not fulfilled for some variables in the model. Additionally, this model is more parsimonious than the non-proportional adjacent category model when some variables violate the proportionality assumption. The parameter estimates and the standard errors in the partial proportional model are feasible, and they can be obtained by fitting a constrained version of the multinomial logistic regression model using the standard maximum likelihood estimation method. The maximum likelihood estimation in the partial proportional adjacent category model can be determined using the VGAM package in R software [33].

The simulation study scenarios are restricted for $R \times C$ tables. We simulated the distribution of the proposed test statistic under the null and alternative hypotheses with 36 and 72 scenarios, respectively. During data generation, we did not consider including any continuous independent variables in the ACM. Thus, the distributional results that are derived from the simulation study are restricted to only categorical independent variables. Possible violations in the chisquare approximation may occur when we add some continuous independent variables in the ACM. Another study to determine the effect of continuous independent variables on the null distribution of the proposed test statistic will be conducted as possible future work.

\section{ACKNOWLEDGEMENTS}

We would like to thank the referee whose suggestions and questions improved the clarity and quality of the paper.

\section{Received 5 September 2013}

\section{REFERENCES}

[1] Hosmer, D. W. and Lemeshow, S., Applied Logistic Regression (2th edn). Wiley, New York, 2000.

[2] Bender, R. and Grouven, U., Using binary logistic regression models for ordinal data with non-proportional odds. Journal of Clinical Epidemiology 1998; 51: 809-816.

[3] NorRis, C. M. et al., Ordinal regression model and the linear regression model were superior to the logistic regression models. Journal of Clinical Epidemiology 2006; 59: 448-456.

[4] Ananth, C. V. and Kleinbaum, D. G., Regression models for ordinal responses: A review of methods and applications. International Journal of Epidemiology 1997; 26: 1323-1333.

[5] Walker, S. H. and Duncan, D. B., Estimation of the probability of an event as a function of several independent variables. Biometrika 1967; 54: 167-79. MR0217928

[6] McCullagh, P., Regression models for ordinal data (with discussion). Journal of the Royal Statistical Society Series B 1980; 42: 109-142. MR0583347

[7] Simon, G., Alternative analyses for the singly-ordered contingency table. Journal of the American Statistical Association 1974; 69: 971-976. MR0428551

[8] Goodman, L. A., The analysis of dependence in crossclassifications having ordered categories, using log-linear models for frequencies and log-linear models for odds. Biometrica 1983; 39: 149-160. MR0712746
[9] Agresti, A., Categorical Data Analysis (2th edn). Wiley, New Jersey, 2002. MR1914507

[10] Fienberg, S. E., The Analysis of Cross-classified Categorical Data. MIT Press, Cambridge, Mass, 1977. MR0464497

[11] McCullagh, P. and Nelder, J. A., Generalized Linear Models (2th edn). Chapman and Hall, London, 1989. MR0727836

[12] Anderson, J. A., Regression and ordered categorical variables (with discussion). Journal of the Royal Statistical Society Series B 1984; 46: 1-30. MR0745211

[13] Engel, J., Polytomous logistic regression. Stat Neerlandica 1988; 42: 233-52. MR0998548

[14] Peterson, B. and Harrell, F. E., Partial Proporional Odds Model for Ordinal Response Variables. Applied Statistics 1990; 2: 205-217.

[15] Greenwood, C. and Farewell, V., A comparison of regression models for ordinal data in an analysis of transplanted-kidney function. The Canadian Journal of Statistics 1988; 16: 325-335.

[16] Holtbrugge, W. and Schumacher, M., A comparison of regression models for the analysis of ordered categorical data. Applied Statistics 1988; 40: 249-259.

[17] Harrell, F. E. et al., Development of a clinical prediction model for an ordinal outcome: The World Health Organization Multicentre Study of Clinical Signs and Etiological Agents of Pneumonia, Sepsis and Meningitis in Young Infants. Statististics in Medicine 1998; 17: 909-944.

[18] LALL, R. et al., A review of ordinal regression models applied on health-related quality of life assessments. Statistical Methods in Medical Research 2002; 11: 49-67.

[19] Imrey, P. B. and Kingman, A., Analysis of clinical trials involving non-cavitated caries lesions. Journal of Dental Research 2004; 83: $103-108$.

[20] Terzi, A. et al., The significance of immunohistochemical expression of Ki-67, p53, p21, and p16 in meningiomas tissue arrays. Pathology - Research and Practice 2008; 204: 305-314.

[21] Long, S. J., Regression Models for Categorical and Limited Dependent Variables, Sage Publications, Thousand Oaks, CA, 1997.

[22] Fu, V. K., Estimating Generalized Ordered Logit Models. Stata Technical Bulletin 44: 27-30. Reprinted in Stata Technical Bulletin Reprints 1998; 8: 160-164.

[23] Williams, R., Generalized ordered logit/partial proportional odds models for ordinal dependent variables. The Stata Journal 2006; 6: 58-82.

[24] Armstrong, B. G. and Slone, M., Ordinal regression models for epidemiologic data. American Journal of Epidemiology 1989; 129: 191-204.

[25] Cole, S. R. and Ananth, C. V., Regression models for unconstrained, partially or fully constrained continuation odds ratios, International Journal of Epidemiology 2001; 30: 1379-1382.

[26] Brant, R., Assessing proportionality in the proportional odds model for ordinal logistic regression. Biometrics 1990; 46: 17711178.

[27] DALe, J. R., Global cross-ratio models for bivariate, discrete and ordered responses. Biometrics 1986; 42: 909-917.

[28] Dos Santos, D. M. and Berridge, D. M., A continuation ratio random effects model for repeated ordinal responses. Statistics in Medicine 2000; 19: 3377-3388.

[29] Wolfe, R., Continuation-ratio models for epidemiological data. Stata Technical Bulletin 1998; 44: 18-21.

[30] Stata Textbook Examples. Applied Logistic Regression, Second Edition, by Hosmer and Lemeshow, Chapter 8: Special Topics. UCLA: Academic Technology Services, Statistical Consulting Group. From http://www.ats.ucla.edu/stat/stata/examples/ alr2/alr2stata8.htm (accessed November 23, 2010).

[31] Jones, B. and Westerland, C., Alternatives to Conventional Practices for Ordinal, Categorical Response Variables. Paper presented at the annual meeting of Midwest Political Science Association, Palmer House Hilton, Chicago, Illinois. From http:// psfaculty.ucdavis.edu/bsjjones/joneswesterland06.pdf (accessed November 23, 2010). 
[32] Wolfe, R. and Gould, W., An Approximate Likelihood-ratio Test for Ordinal Response Models. Stata Technical Bulletin 1998; 42: 24-27.

[33] Yee, T. W., The VGAM package for categorical data analysis. Journal of Statistical Software 2010; 32: 1-34.

[34] Serfling, R. J., Approximation Theorems of Mathematical Statistics. Wiley, New York, 1980. MR0595165

Anil Dolgun

Hacettepe University Faculty of Medicine

Department of Biostatistics

Sihhiye 06100

Ankara

Turkey

E-mail address: anilbarak@yahoo.com
Osman Saracbasi

Hacettepe University Faculty of Medicine

Department of Biostatistics

Sihhiye 06100

Ankara

Turkey

E-mail address: osaracba@hacettepe.edu.tr 